\title{
Gravitational wave echo of relaxion trapping
}

\author{
Abhishek Banerjee $\odot,{ }^{1, *}$ Eric Madge $\odot,{ }^{1, \dagger}$ Gilad Perez, ${ }^{1, \star}$ Wolfram Ratzinger, ${ }^{2, \S}$ and Pedro Schwaller ${ }^{2, \|}$ \\ ${ }^{1}$ Department of Particle Physics and Astrophysics, Weizmann Institute of Science, Rehovot 7610001, Israel \\ ${ }^{2}$ PRISMA $^{+}$Cluster of Excellence and Mainz Institute for Theoretical Physics, \\ Johannes Gutenberg-Universität Mainz, 55099 Mainz, Germany
}

(Received 16 June 2021; accepted 27 July 2021; published 21 September 2021)

\begin{abstract}
To solve the hierarchy problem, the relaxion must remain trapped in the correct minimum, even if the electroweak symmetry is restored after reheating. In this scenario, the relaxion starts rolling again until the backreaction potential, with its set of local minima, reappears. Depending on the time of barrier reappearance, Hubble friction alone may be insufficient to retrap the relaxion in a large portion of the parameter space. Thus, an additional source of friction is required, which might be provided by coupling to a dark photon. The dark photon experiences a tachyonic instability as the relaxion rolls, which slows down the relaxion by backreacting to its motion, and efficiently creates anisotropies in the dark photon energy-momentum tensor, sourcing gravitational waves. We calculate the spectrum of the resulting gravitational wave background from this new mechanism and evaluate its observability by current and future experiments. We further investigate the possibility that the coherently oscillating relaxion constitutes dark matter and present the corresponding constraints from gravitational waves.
\end{abstract}

DOI: 10.1103/PhysRevD.104.055026

\section{INTRODUCTION}

The weakness of the gravitational force ensures that gravitational waves (GWs) can store information about the history of our Universe. On the other hand, though, it also implies that only dramatic and cosmological events may leave imprints that can be observed at present or in the near future. GW detectors are, for instance, sensitive to primordial phase transitions, inflation, and oscillatory motion of ultralight fields (see, e.g., Refs. [1,2] for a recent discussion of potential signals of cosmological origin).

In this work, we introduce a different mechanism that leads to the production of GWs in the early Universe. In the following, we demonstrate the idea using the relaxion framework [3]; however, the core scheme may be applicable to other forms of new physics. At early times, the relaxion is trapped in a local minimum. However, assuming that the reheating temperature is above the electroweak (EW)

\footnotetext{
*abhishek.banerjee@weizmann.ac.il

†eric.madge-pimentel@weizmann.ac.il

*gilad.perez@weizmann.ac.il

\$.ratzinger@uni-mainz.de

" pedro.schwaller@uni-mainz.de
}

Published by the American Physical Society under the terms of the Creative Commons Attribution 4.0 International license. Further distribution of this work must maintain attribution to the author(s) and the published article's title, journal citation, and DOI. Funded by SCOAP ${ }^{3}$. scale, ${ }^{1}$ we expect the EW symmetry to be restored. Hence, the minimum in which the relaxion was originally trapped disappears, and the relaxion starts rolling down its potential. At some temperature below the EW scale, we expect the set of local minima to reappear. There is a limited region of the model's parameter space such that the relaxion would be trapped still within the same minimum [6,7]. In this case, even the minimal realization of the relaxation mechanism could lead to a viable ultralight dark matter (DM) candidate [6], which may be tested in the future due to the presence of relaxion-Higgs mixing [8,9].

Despite the attractiveness of this minimal setup, there is a sizeable region of parameter space where the postreheating displacement of the relaxion would be too large and the relaxion would not be trapped but instead would descend in an uncontrolled way toward the global minimum. This runaway can be avoided if an additional source of friction is added to the model. Coupling a scalar field to a dark $U(1)$ gauge field strength is known to lead to an efficient energy loss mechanism in the form of tachyonic dark photon field production [10,11] (see Refs. [12,13] for the case of a relaxion or other axionlike particles (ALPs) in the mass range $\gtrsim \mathcal{O}(\mathrm{MeV})$, where self-friction may be induced, or

\footnotetext{
${ }^{1}$ The requirement of a high reheating temperature, motivated by a large class of inflation models (see, e.g., Ref. [4] and references therein), is generic and also needed in most models that explain the observed baryon abundance (see, e.g., Ref. [5] and references therein).
} 
Ref. [14] for stopping via an instability in a modified relaxion potential, albeit outside the region of interest of this work). Dark photon production quickly reaches a quasisteady-state where the friction balances the slope of the potential. At each time, the dominantly produced momentum-mode $k=\xi a H$ is about to exit the tachyonic band, where $\xi$ is an $\mathcal{O}(10-100)$ parameter, $H$ is the Hubble rate, and $a$ is the scale factor.

The energy density stored in this mode is roughly constant, $\rho_{X} \sim m_{\phi}^{2} f_{\phi}^{2}[6]$, where $m_{\phi}$ and $f_{\phi}$ are the relaxion mass and the decay constant, respectively, and is the source of GW production. The rolling of the relaxion and the dark photon production stop around the time when the potential barriers reappear, which traps the relaxion and ends this epoch. ${ }^{2}$

Given the above mechanism, we can estimate the GW signal as follows. First, the peak frequency at present time is obtained by redshifting the dominant $k$ mode at the time of reappearance, cf. Eq. (35),

$$
f_{\text {peak }} \sim \frac{a_{\mathrm{ra}}}{a_{0}} \xi H_{\mathrm{ra}} \sim 1 \mu \mathrm{Hz}\left(\frac{\xi}{25}\right)\left(\frac{T_{\mathrm{ra}}}{1 \mathrm{GeV}}\right),
$$

where $T_{\mathrm{ra}}$ is the temperature when the potential barriers reappear. Here and in the following, quantities indexed "0" and "ra" are evaluated today and at the time when the potential barriers reappear, respectively. Second, the GW amplitude is roughly given by the square of the energy density stored in the dark photon just before $T_{\text {ra }}$, cf. Eq. (28),

$$
\begin{aligned}
\Omega_{\mathrm{GW}}^{\mathrm{peak}} & \sim \frac{1}{\rho_{c}^{0}} \frac{\left(c_{\mathrm{eff}} \rho_{X}^{\mathrm{ra}} / f_{\mathrm{peak}}^{\mathrm{ra}}\right)^{2}}{M_{\mathrm{Pl}}^{2}}\left(\frac{a_{\mathrm{ra}}}{a_{0}}\right)^{4} \\
& \sim 10^{-10}\left(\frac{25}{\xi}\right)^{2}\left(\frac{m_{\phi}}{0.1 \mathrm{eV}} \frac{f_{\phi}}{10^{10} \mathrm{GeV}}\right)^{4}\left(\frac{1 \mathrm{GeV}}{T_{\mathrm{ra}}}\right)^{8} \\
& \sim 10^{-12}\left(\frac{25}{\xi}\right)^{2}\left(\frac{0.1 \mathrm{eV} \sin \theta_{\phi h}}{m_{\phi}} \frac{12}{10^{-13}}\right)^{12}\left(\frac{\mathrm{TeVGVV}}{\Lambda} \frac{\mathrm{GeV}}{T_{\mathrm{ra}}}\right)^{8},
\end{aligned}
$$

where $\rho_{c}^{0}=3 M_{\mathrm{Pl}}^{2} H_{0}^{2}$ is the critical energy density of the Universe today, $f_{\text {peak }}^{\text {ra }}=\xi H_{\text {ra }}$ is the peak frequency without red-shifting, and $c_{\text {eff }}^{2} \simeq 1 / 4$ is the efficiency factor for converting dark photon energy into GWs obtained from the analytic calculation in the Appendix C. Note that $c_{\text {eff }} \sim$ $\mathcal{O}(1)$ as the relaxion potential energy at each time is predominantly deposited in a narrow range of exponentially growing dark photon momentum modes, resulting in large time-dependent inhomogeneities and an efficient generation of anisotropic stress sourcing GWs, in a similar manner as in the case of tachyonic preheating [16-19]. In the last line, we have reexpressed the signal strength in terms of the

\footnotetext{
${ }^{2}$ For simplicity, we are agnostic about the details of the reappearance process of the backreaction potential and assume instantaneous reappearance. Various considerations regarding barrier reappearance can also generate gravitational waves, a scenario considered in Ref. [15].
}

relaxion-Higgs mixing $\sin \theta_{h \phi}$ and the cutoff scale $\Lambda$. This result shows that some of the parameter space of the model may lead to a visible signal in near-future $\mathrm{GW}$ experiments, allowing us to probe parameter regions that are currently unexplored by other experiments, as discussed below. In addition, we note that the relation between the physical parameters of the models and the GW amplitude is given by $\sin \theta_{h \phi} \propto m_{\phi} \times\left(\Omega_{\mathrm{GW}}^{\mathrm{peak}}\right)^{1 / 12}$, showing a rather mild dependence on the actual amplitude.

For convenience, the range of relaxion masses, $m_{\phi}$, and mixing angles with the Higgs boson, $\sin \theta_{h \phi}$, which can be probed via current or future GW experiments as well as the corresponding constraints on the parameter space are summarized in Fig. 1. The green and blue/turquoise colored regions can be accessed with $\mu$ Ares and the SquareKilometre Array (SKA) observatory, respectively, depending on the temperature of barrier reappearance. In the purple region, the reappearance temperature is restricted by current data from the North American Nanohertz Observatory for Gravitational Waves (NANOGrav). In addition to that, in the gray shaded region, we present the parameter range in which our model can account for the potential GW signal recently observed in NANOGrav data. The gray dashed line encloses the region in which the relaxion can be trapped without dark photon friction. On the other hand, as the figure illustrates, there is a large fraction of parameter space where an additional source of friction is required for the viability of the relaxion mechanism and thus motivates us to add a relaxion-dark photon coupling to the model. A more detailed discussion of the figure is deferred to Sec. IV.

This paper is organized as follows. We briefly review the relaxion and the dark photon dynamics in Sec. II. Sections II A and II B contain a brief discussion of the interplay of the relaxion-dark photon dynamics and the possibility of the relaxion being DM, respectively. In Sec. II C, we review the constraints on our model and discuss the available parameter space. Subsequently, the production mechanism of the GW background is studied in Sec. III. We derive the GW spectrum in Sec. III A and briefly describe how the detectability of the signal is evaluated in Sec. III B. The results of this paper are then discussed in Sec. IV. Section V concludes the paper. A brief discussion of the minimal relaxion scenario (without a dark photon coupling) is deferred to Appendix A. Further details regarding the calculation of the dark photon and GW spectra are provided in Appendixes B and C, respectively.

\section{SETUP}

In this work, we consider the relaxion $\phi$ coupled to a dark photon field $X_{\mu}$,

$$
-\mathcal{L} \supset V(H, \phi)+\frac{r_{X}}{4} \frac{\phi}{f_{\phi}} X_{\mu \nu} \tilde{X}^{\mu \nu},
$$




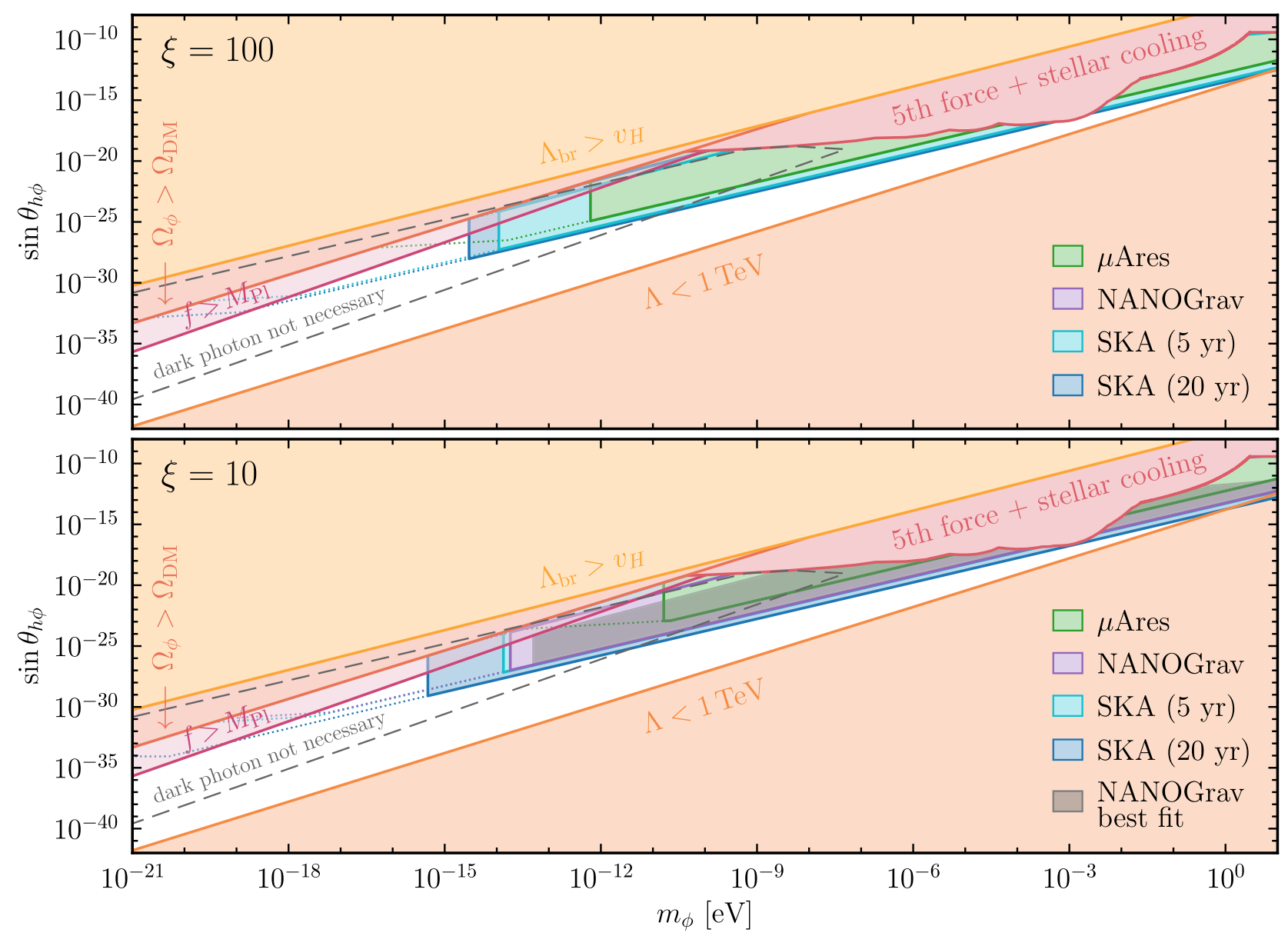

FIG. 1. Available parameter space for $\xi=100$ (top), and for $\xi=10$ (bottom). The red and orange shaded regions are excluded by the indicated constraints or combinations thereof. Above the red solid line, the relaxion decay constant becomes super-Planckian. The gray dashed line encloses the parameter space in which relaxation can be realized without dark photon friction, which is discussed in more details in Appendix A. The prospective GW sensitivity of $\mu$ Ares (green) as well as SKA after an observation period of 5 years (turquoise) and 20 years (blue) is indicated by the respective colored regions. In the purple colored region, a subrange of the viable reappearance temperatures can be excluded using current NANOGrav data from the 11 year dataset. The regions bounded by the colored dotted lines need super-Planckian decay constants to be probed by the respective experiment. In the lower panel, the gray shaded region can reproduce our best-fit spectrum (at $T_{\mathrm{ra}} \sim 20 \mathrm{MeV}$ ) to the potential stochastic GW background seen in the recent NANOGrav data. An animated version of the lower panel of the figure, explicitly showing the dependence on the reappearance temperature, can be found in the Supplemental Material [20] of this paper.

with the potential of the relaxion field $\phi$ and Higgs doublet $H$ given by

$V(H, \phi)=V_{\mathrm{roll}}(\phi)+\mu_{H}^{2}(\phi)|H|^{2}+\lambda|H|^{4}+V_{\mathrm{br}}(H, \phi)$,

where $\lambda$ is the Higgs quartic coupling and

$$
\begin{aligned}
V_{\text {roll }}(\phi) & =-c g \Lambda^{3} \phi, \\
\mu_{H}^{2}(\phi) & =\Lambda^{2}-g \Lambda \phi, \\
V_{\mathrm{br}}(H, \phi) & =-\frac{\Lambda_{\mathrm{br}}^{4}}{v_{H}^{2}}|H|^{2} \cos \frac{\phi}{f_{\phi}} .
\end{aligned}
$$

Here, $c$ is an $\mathcal{O}(1)$ number, $g$ is a dimensionless parameter, $\Lambda$ is the Higgs mass cutoff scale, $\Lambda_{\mathrm{br}}$ is the backreaction scale, $v_{H}=\langle|H|\rangle=174 \mathrm{GeV}$ is the Higgs vacuum expectation value, and $f_{\phi}$ is the decay constant of the relaxion.

During inflation, the relaxion rolls down the linear slope of its potential $V_{\text {roll }}$. It thereby scans the Higgs mass parameter $\mu^{2}(\phi)$. Once $\mu^{2}$ crosses zero, the Higgs acquires a nonvanishing vacuum expectation value, triggering the breaking of the EW gauge symmetry. The Higgs then backreacts, creating wiggles in the relaxion potential via $V_{\mathrm{br}}$. Once the Higgs backreaction balances the rolling potential, the relaxion is trapped in the first minimum it encounters. Choosing $c g \Lambda^{3} f_{\phi} \sim \Lambda_{\mathrm{br}}^{4}$, we end up with a weak-scale expectation value for the Higgs boson, solving the hierarchy problem. The relaxion mass and the relaxionHiggs mixing angle can then be written as [7,9] 


$$
m_{\phi}^{2} \simeq \frac{\Lambda_{\mathrm{br}}^{6}}{f_{\phi}^{2} \Lambda v_{H}}, \quad \sin \theta_{h \phi} \simeq \sqrt{2}\left(\frac{m_{\phi}^{4} f_{\phi} \Lambda^{2}}{v_{H} m_{h}^{6}}\right)^{\frac{1}{3}},
$$

in terms of the theory parameters. Here, $m_{h}=125 \mathrm{GeV}$ is the Higgs mass.

\section{A. Relaxion and dark photon evolution}

After reheating, the EW symmetry will be restored due to thermal corrections to the potential, provided that the reheating temperature is above the EW phase transition temperature. As a consequence, the relaxion will start rolling again, leading to exponential production of dark photon modes. To see the interplay, the coupled differential equations describing the evolution of the spatially homogeneous relaxion and the dark photon modes are given by

$$
\begin{gathered}
\theta^{\prime \prime}+2 a H \theta^{\prime}+\frac{a^{2}}{f_{\phi}^{2}} \frac{\partial V_{\text {roll }}}{\partial \theta}=-\frac{a^{2}}{f_{\phi}^{2}} \frac{r_{X}}{4 a^{4}}\left\langle X_{\mu \nu} \tilde{X}^{\mu \nu}\right\rangle, \\
X_{\lambda}^{\prime \prime}(k, \tau)+\left(k^{2}-\lambda k r_{X} \theta^{\prime}\right) X_{\lambda}(k, \tau)=0,
\end{gathered}
$$

where $\theta=\phi / f_{\phi}$ and primes denote derivatives with respect to conformal time $\tau$ with $a d \tau=d t$. We have written the dark photon in terms of its Fourier modes $X_{\lambda}(\mathbf{k}, \tau)$ in Coulomb gauge, $\nabla \cdot \mathbf{X}=0$, as

$$
\hat{\mathbf{X}}(\mathbf{x}, \tau)=\int \frac{d^{3} k}{(2 \pi)^{3}} \sum_{\lambda= \pm} X_{\lambda}(k, \tau) \varepsilon_{\lambda}(\mathbf{k}) \hat{a}_{\lambda}(\mathbf{k})+\text { H.c. }
$$

Here, $\lambda$ denotes the dark photon helicity, and $\varepsilon_{\lambda}$ are the corresponding circular polarization vectors.

As evident from Eq. (8), dark photon modes with $0<k<\lambda r_{X} \theta^{\prime}$ are tachyonic and will experience exponential growth compared to the vacuum fluctuations. The resulting dark photon spectrum then features anisotropies in its energy-momentum tensor which will act as a source for GW production, leading to a stochastic GW background [17-19,21-23]. Furthermore, since only modes of the helicity with the same sign as the relaxion velocity can become tachyonic, the rolling relaxion will produce a circular polarized dark photon background. In our case, as we choose $\theta^{\prime}>0$, only the positive-helicity modes are exponentially produced. For these modes, the solution to the equations of motion is given in the WentzelKramers-Brillouin (WKB) approximation by

$$
X_{+}(k, \tau)=\frac{e^{g(k, \tau)}}{\sqrt{2 \Omega(k, \tau)}},
$$

where $\Omega^{2}(k, \tau)=k r_{X}\left|\theta^{\prime}(\tau)\right|-k^{2}>0$ is the corresponding tachyonic frequency and $g(k, \tau)=\int{ }^{\tau} d \tau^{\prime} \Omega\left(k, \tau^{\prime}\right)$. The approximation holds for $\left|\Omega^{\prime} / \Omega^{2}\right| \ll 1$.
At early times, just after reheating, the friction from dark photons can be neglected. Assuming radiation domination, the relaxion velocity can then be written as

$$
\theta^{\prime}(\tau)=\frac{\Lambda_{\mathrm{br}}^{4}}{5 f_{\phi}^{2}}\left(\frac{a_{\mathrm{rh}}}{\tau_{\mathrm{rh}}}\right)^{2} \tau^{3}\left[1-\left(\frac{\tau_{\mathrm{rh}}}{\tau}\right)^{5}\right],
$$

where we imposed $\theta^{\prime}\left(\tau_{\mathrm{rh}}\right)=0$. Subsequently, due to the exponential production, the dark photon friction becomes comparable to the other terms in the equation of motion, Eq. (7), and asymptotes to the slope of the rolling potential. The timescale $\tau_{\mathrm{pp}}$ at which particle production kicks in can be determined by

$$
\frac{\left\langle X_{\mu \nu} \tilde{X}^{\mu \nu}\right\rangle\left(\tau_{\mathrm{pp}}\right)}{4 a^{4}\left(\tau_{\mathrm{pp}}\right)} \approx \frac{\tilde{k}^{4} e^{2 g(\tilde{k} \tilde{\tau})}}{4 \pi^{2} a^{4}} \sim \frac{\Lambda_{\mathrm{br}}^{4}}{r_{X}},
$$

where $\tilde{k}$ is the mode that dominates the $\langle X \tilde{X}\rangle$ term, given by the saddle point $\partial g(k, \tau) /\left.\partial k\right|_{\tilde{k}}=0$. After $\tau_{\mathrm{pp}}$, due to the balance between the potential slope and the backreaction from the dark photon, the relaxion field velocity becomes proportional to the Hubble rate and evolves as $[6,10]$

$$
\theta^{\prime}(\tau) \approx \frac{\xi}{r_{X}} a(\tau) H(\tau)\left(1+\epsilon \log \frac{\tau}{\tau_{\mathrm{pp}}}\right) \approx \frac{\xi}{r_{X} \tau},
$$

with a small logarithmic correction $(\epsilon \ll 1)$. We defined here the parameter $\xi=\frac{r_{X}\left|\theta^{\prime}\right|}{a H}$ at $\tau_{\mathrm{pp}}$. From Eq. (12), we then obtain $\xi \sim \mathcal{O}(10-100)$ with a mere logarithmic dependence on the relaxion parameters [10]. The dominating $k$ mode at each epoch then becomes $\tilde{k} / a \sim r_{X} \dot{\theta}(t) \sim \xi H$.

Once the Universe has cooled sufficiently, the EW phase transition occurs, and the wiggles of the backreaction potential reappear. The rolling of the relaxion between reheating and $T_{\text {ra }}$ leads to a displacement from the minimum in which it originally settled during inflation by

$$
\Delta \theta=\int_{\tau_{\mathrm{rh}}}^{\tau_{\mathrm{ra}}} d \tau \theta^{\prime} \approx \frac{\xi}{4 r_{X}}\left[1+\log \frac{H_{\mathrm{pp}}^{2}}{H_{\mathrm{ra}}^{2}}\right] .
$$

For the relaxion to remain trapped in this minimum, we need to require that the displacement is less than the distance between the minimum and the next maximum, $\Delta \theta \lesssim 2 \delta$, where $\delta=\Lambda_{\mathrm{br}}^{2} /\left(\Lambda v_{H}\right)$ [6,7]. This sets a lower bound on the coupling to the dark photons. For smaller couplings, the dark photon friction is insufficient to prevent the relaxion from rolling into one of the neighboring minima. The relaxion then needs to traverse $\Delta \theta \sim \mathcal{O}(n)$ to end up in the $n$-th minimum, where $n=1$ denotes the minimum in which it stopped during inflation, extending the parameter space of the theory. However, going beyond the first minimum requires a careful adjustment of the initial conditions to let the relaxion stop exactly at the bottom of the $n$th minimum at reappearance. Otherwise, 
the time required for the relaxion to reach the bottom would exceed the age of the Universe. We thus simply assume $r_{X}=\xi /(2 \delta)$ in the following. ${ }^{3}$

\section{B. Relaxion dark matter}

After the reappearance of the Higgs backreaction potential, the displaced relaxion begins to oscillate around the minimum of its potential, providing a candidate for ultralight DM as discussed in Ref. [6]. Assuming the maximal displacement of $\Delta \theta=2 \delta$, the relaxion relic abundance is given by

$$
\Omega_{\phi}=\frac{2 m_{\phi}^{2} f_{\phi}^{2} \delta^{2}}{3 M_{\mathrm{Pl}}^{2} H_{0}^{2}} \frac{g_{* s}\left(T_{0}\right) T_{0}^{3}}{g_{* s}\left(T_{\mathrm{osc}}\right) T_{\mathrm{osc}}^{3}},
$$

where $T_{\text {osc }} \sim \min \left[T_{\mathrm{ra}}, \sqrt{m_{\phi} M_{\mathrm{Pl}}}\right]$ is the temperature when the relaxion starts to oscillate. Requiring that the relaxion provides all of DM, i.e., $\Omega_{\phi} h^{2}=0.12$ [28], the relaxion decay constant can be expressed as

$f_{\phi} \sim 5 \times 10^{9} \mathrm{GeV}\left(\frac{\Lambda}{1 \mathrm{TeV}}\right)^{\frac{2}{5}}\left(\frac{T_{\mathrm{osc}}}{1 \mathrm{GeV}}\right)^{\frac{9}{10}}\left(\frac{0.1 \mathrm{eV}}{m_{\phi}}\right)$.

As we are considering coherently oscillating relaxion DM here, its mass needs to be less than approximately $10 \mathrm{eV}$ in order to be described by a classical field [29]. For this range of relaxion masses, the possible decay channels are into two photons and two dark photons, $\Gamma_{\phi}=\Gamma_{\gamma \gamma}+\Gamma_{X X}$. The decay rate into a dark photon pair is given by

$$
\Gamma_{X X}=\frac{r_{X}^{2}}{64 \pi} \frac{m_{\phi}^{3}}{f_{\phi}^{2}},
$$

while the decay width of $\phi \rightarrow \gamma \gamma$ is subdominant compared to that of $\phi \rightarrow X X$ as it is suppressed by the square of the relaxion-Higgs mixing angle [6], which in turn is bounded from above by $\sin \theta_{h \phi} \lesssim v_{H} / f_{\phi}$ [9]. The relaxion lifetime hence becomes

$$
\tau_{\phi} \sim 20 \mathrm{Gyr}\left(\frac{25}{\xi}\right)^{2}\left(\frac{T_{\mathrm{osc}}}{1 \mathrm{GeV}}\right)^{3}\left(\frac{0.1 \mathrm{eV}}{m_{\phi}}\right)^{5}
$$

where we have chosen $r_{X}=\xi /(2 \delta)$. Since the decay of DM into relativistic particles affects the spectrum of the cosmic microwave background $(\mathrm{CMB})$ at low- $\ell$ multipoles, the lifetime is constrained as $\tau_{\phi}>160 \mathrm{Gyr}$ [30].

As shown in Refs. [23,31-33], for $r_{X}(\Delta \theta)_{\text {sep }} \sim \mathcal{O}\left(10^{2}\right)$, an oscillating ALP may introduce a second phase of tachyonic dark photon production, which could suppress

\footnotetext{
${ }^{3}$ As $\delta=\frac{\Lambda_{\mathrm{br}}^{2}}{\Lambda v_{H}}<\frac{v_{H}}{\Lambda}$, this implies $r_{X} \gtrsim 10^{2}\left(\frac{\Lambda}{1 \mathrm{TeV}}\right)\left(\frac{\xi}{10}\right)$. Such large couplings can be obtained in a technically natural way, for example, via the clockwork mechanism [24-27].
}

the DM abundance by up to 2 orders of magnitude. This condition is satisfied in parts of the parameter space where the field displacement is maximal, since there $r_{X} \sim \mathcal{O}(\xi / \delta)$ and $\xi \sim \mathcal{O}\left(10-10^{2}\right)$, and introduces some uncertainty in our estimate of the DM abundance in those regions.

\section{Constraints}

A successful cosmological relaxation of the Higgs mass requires the inflation sector to dominate the total energy density, $3 H_{\mathrm{I}}^{2} M_{\mathrm{Pl}}^{2} \gtrsim \Lambda^{4}$, as well as that the classical motion of the relaxion dominates over quantum fluctuation during inflation, $(\Delta \phi)_{\mathrm{cl}} \gtrsim H_{\mathrm{I}} / 2 \pi$. Here, $H_{\mathrm{I}}$ is the Hubble scale during inflation. Combining these two constraints, we get an upper bound on the cutoff scale $\Lambda$,

$$
\Lambda \lesssim\left(\frac{2 \pi \sqrt{3} M_{\mathrm{Pl}}^{3} \Lambda_{\mathrm{br}}^{4}}{f}\right)^{1 / 6}
$$

As we are considering a Higgs-dependent backreaction potential, we also require $\Lambda_{\mathrm{br}} \lesssim v_{H}[3,9]{ }^{4}$ The allowed range of the effective cutoff $\Lambda$ of the theory is

$$
M_{\mathrm{Pl}} \gtrsim f_{\phi} \gtrsim \Lambda \gtrsim 4 \pi v_{H} \sim 1 \mathrm{TeV} .
$$

Also, for a generic backreaction potential which does not change the late-time cosmology, the range of reappearance temperatures is

$$
v_{H} \gtrsim T_{\mathrm{ra}} \gtrsim T_{\mathrm{BBN}} \sim 10 \mathrm{MeV} .
$$

For masses below the eV scale, the relaxion can further mediate long-range forces. Experiments looking for such interactions (fifth force experiments, inverse-square-law, and equivalence-principle tests) constrain the coupling of the relaxion to ordinary matter [34-40], which is induced by the relaxion-Higgs mixing angle given in Eq. (6). In a similar manner, for masses up to the $\mathrm{keV}$ range, the mixing is constrained from stellar cooling [41-44], as it induces relaxion-mediated contributions to the energy loss in stars. Slightly weaker limits on the mixing angle can furthermore be obtained from bounds on the solar relaxion flux as constrained by XENON1T and other dark-matter direct detection experiments [45].

Additional constraints arise when coupling the relaxion to a dark photon field (see Appendix A for a discussion of the minimal scenario without this coupling), with the coupling here chosen to saturate the trapping condition, i.e., $r_{X}=\xi /(2 \delta)$. For the dark photon induced friction to trap the relaxion, reappearance has to occur sufficiently late for the dark photon to be produced, i.e., $H_{\mathrm{pp}}>H_{\mathrm{ra}}$. This

\footnotetext{
${ }^{4}$ See Refs. [11,13] for a discussion of other stopping mechanisms in which this constraint can be relaxed.
} 
sets a lower bound on the relaxion mass, $m_{\phi} \gtrsim \sqrt{10} \delta H_{\text {ra }}$, for which the dark photon scenario can be applied.

If this condition is satisfied, throughout its evolution from reheating to reappearance, the relaxion continuously produces dark photons, depositing energy density into the latter. At the time of trapping, $t_{\mathrm{ra}}$, the dark photon energydensity can be estimated as

$$
\rho_{X}\left(t_{\mathrm{ra}}\right)=\frac{1}{a^{4}\left(t_{\mathrm{ra}}\right)} \int^{t_{\mathrm{ra}}} d t^{\prime} a^{4}\left(t^{\prime}\right) \dot{V}=\frac{1}{2} m_{\phi}^{2} f_{\phi}^{2}
$$

For the Universe to remain radiation dominated, we require $\rho_{X}\left(T_{\text {ra }}\right)$ to be smaller than the radiation energy density at $T_{\text {ra }}$, i.e., $\rho_{X}\left(T_{\text {ra }}\right) \lesssim 3 M_{\mathrm{Pl}}^{2} H_{\mathrm{ra}}^{2} \sim T_{\mathrm{ra}}^{4}$; otherwise, the relaxion dominates the total energy density of the Universe. After $T_{\text {ra }}$, dark photon production stops, and the corresponding energy density redshifts as that of radiation.

The dark photon is further constrained by its contribution to the energy density of the Universe at low temperatures, typically parametrized in terms of the effective number of neutrino species $N_{\text {eff }}$. For temperatures $T \lesssim m_{e}$, after electrons and positrons have annihilated, the dark photon energy density (or of any additional relativistic species for that matter) can be written in terms of its contribution $\Delta N_{\text {eff }}$ to $N_{\text {eff }}$ as

$$
\rho_{X}(T)=\frac{7 \pi^{2}}{120} \Delta N_{\text {eff }}\left(\frac{4}{11}\right)^{\frac{4}{3}} T^{4},
$$

where the 4/11 factor accounts for the difference between the neutrino and photon temperature. Using Eq. (22) and the current $95 \%$ C.L. limit $N_{\text {eff }}=3.27 \pm 0.29$ from CMB data [28] combined with local measurements of the Hubble rate [46], as well as $N_{\text {eff }}^{\mathrm{SM}}=3.046$ in the Standard Model, we obtain a lower limit on the reappearance temperature as a function of the relaxion mass and decay constant

$$
T_{\text {ra }} \gtrsim 2.5 g_{* s, \text { ra }}^{-1 / 3} \sqrt{m_{\phi} f_{\phi}},
$$

where $g_{* s, \text { ra }}$ is the number of entropic degrees of freedom at reappearance.

If we assume that the relaxion accounts for the full DM abundance, then plugging Eq. (16) to Eq. (24), we get

$$
T_{\mathrm{ra}} \gtrsim 450 \mathrm{MeV}\left(\frac{67}{g_{* s, \mathrm{ra}}}\right)^{\frac{1}{3}}\left(\frac{\Lambda}{10 \mathrm{TeV}}\right)^{\frac{4}{11}} .
$$

Here, we also assume that the relaxion starts to oscillate at $T_{\text {ra }}$, which is only true for a sufficiently heavy relaxion. As we require $\Lambda \gtrsim 4 \pi v_{H} \sim 1 \mathrm{TeV}$, this sets a lower bound of $T_{\text {ra }} \gtrsim 240 \mathrm{MeV}$ on the reappearance temperature for the

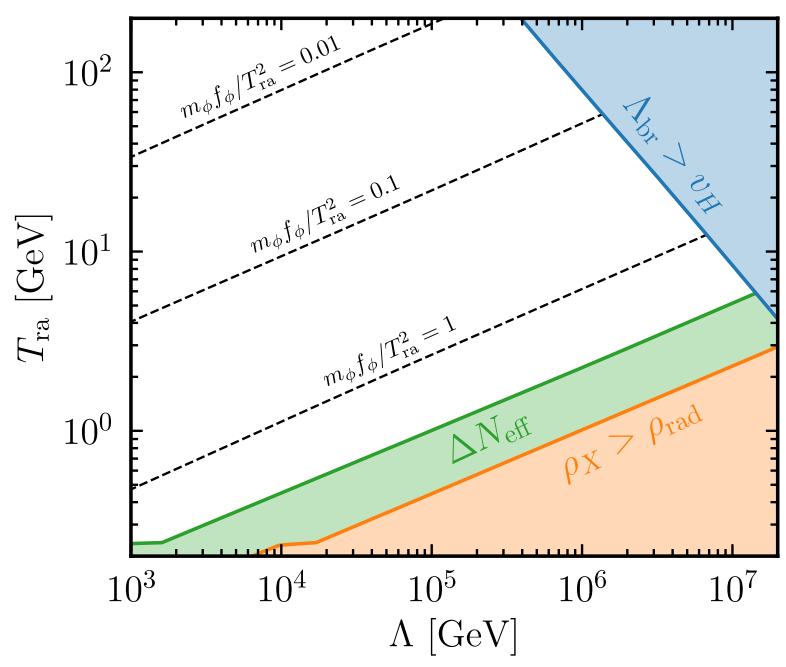

FIG. 2. Allowed range of reappearance temperatures $T_{\text {ra }}$ as a function of the cutoff scale $\Lambda$, while fixing $f_{\phi}$ to the value reproducing the measured DM abundance, Eq. (16), assuming $T_{\text {osc }}=T_{\text {ra }}$. The blue shaded region is excluded since $\Lambda_{\mathrm{br}}>v_{H}$, while in the green shaded regions, the CMB bound on $\Delta N_{\text {eff }}$, Eq. (25), is violated. In the orange shaded region, the dark photon energy density further dominates the Universe at reappearance. The dashed lines are contours of constant $m_{\phi} f_{\phi} / T_{\text {ra }}^{2}$, which sets the amplitude of the GW spectrum; cf. Eq. (2).

relaxion DM scenario to be realized. ${ }^{5}$ Upon the same assumptions, $\Lambda_{\mathrm{br}} \lesssim v_{H}$ further sets an upper bound on the reappearance temperature,

$$
T_{\text {ra }} \lesssim \min \left[v_{H}, 80 \mathrm{GeV}\left(\frac{10^{6} \mathrm{GeV}}{\Lambda}\right)\left(\frac{96}{g_{* \mathrm{~s}, \mathrm{ra}}}\right)^{\frac{1}{3}}\right] .
$$

In Fig. 2, we show the minimal and maximal allowed reappearance temperature for relaxion DM as a function of the cutoff $\Lambda$ of the theory. Combining $\Lambda_{\mathrm{br}}<v_{H}$ (blue) and the $N_{\text {eff }}$ constraint (green), we see that the highest $\Lambda$ for which the relaxion can be realized as coherently oscillating $\mathrm{DM}$ is $\Lambda \lesssim 10^{7} \mathrm{GeV}$, which is in accordance with the constraints for $T_{\text {ra }} \simeq 6 \mathrm{GeV}$. Because of the rapid change in the radiative degrees of freedom around the time of the QCD phase transition, the $\Delta N_{\text {eff }}$ limit on the reappearance temperature saturates at $T_{\mathrm{ra}} \sim T_{\mathrm{QCD}}$ for $\Lambda \lesssim 2 \mathrm{TeV}$. We also depict the weaker bound $\rho_{X}<\rho_{\text {rad }}$ from the total energy density in orange.

In Fig. 3, we depict the constraints on the relaxion parameters as a function of the relaxion mass $m_{\phi}$ and the mixing angle $\sin \theta_{h \phi}$, where we determined $\Lambda$ and $f_{\phi}$ from the DM abundance using Eqs. (6) and (16). The red and orange shaded regions are excluded by the indicated constraints, where the shape of the exclusions now partially

\footnotetext{
${ }^{5}$ Note that, for this value of the reappearance temperature, the relaxion starts to oscillate directly after reappearance as long as $m_{\phi} \gtrsim 5 \times 10^{-10} \mathrm{eV}$.
} 


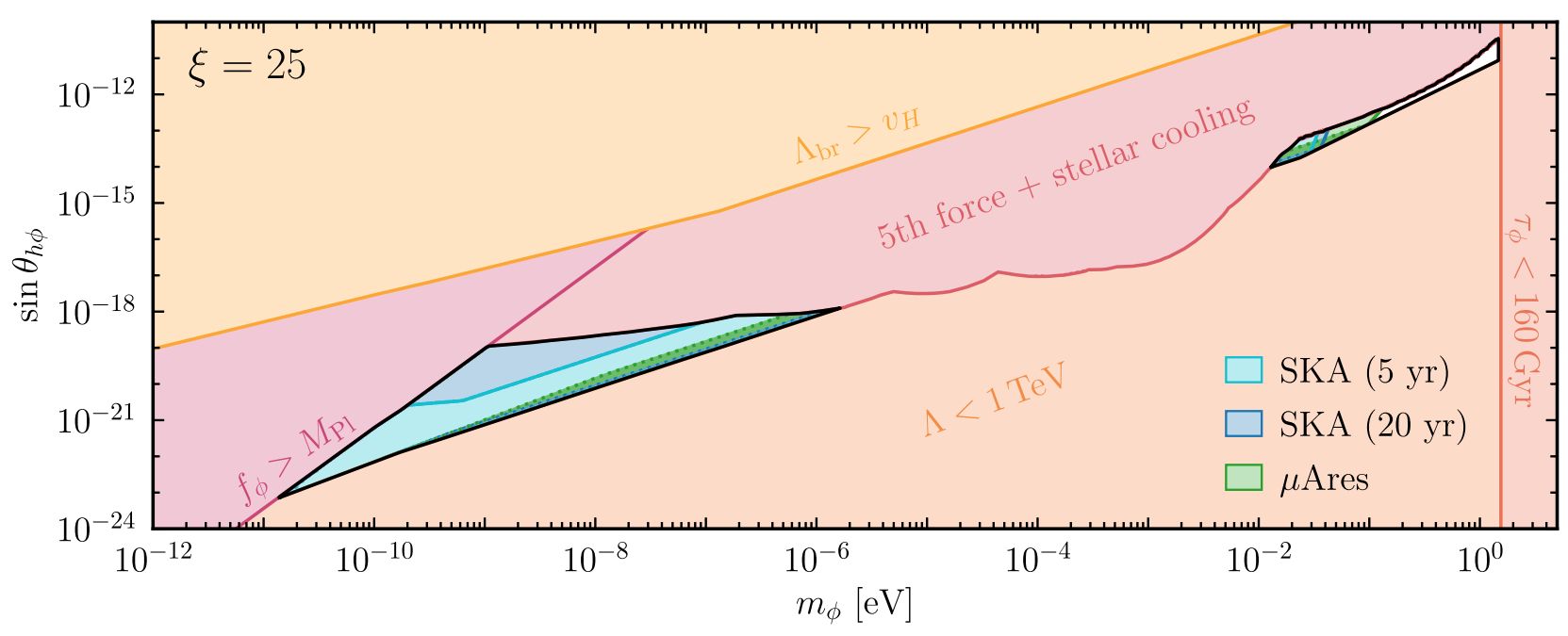

FIG. 3. Available parameter space (black framed region) for relaxion DM in the relaxion mass $m_{\phi}$ vs mixing angle sin $\theta_{h \phi}$ plane. The red and orange shaded regions are excluded by the indicated constraints of combinations thereof. The colored regions inside the viable DM space can be probed via GWs in $\mu$ Ares (green) or SKA (blue/turquoise). The light shading and solid lines indicate points that can be probed for a subrange of reappearance temperatures, whereas the darker shaded parts enclosed by dotted lines are accessible for all valid $T_{\text {ra }}$. An animated version of the plot scanning the reappearance temperature is enclosed in the Supplemental Material [20] of this work.

differs from the corresponding ones in Fig. 1 as we require here $\Omega_{\phi}=\Omega_{\mathrm{DM}}$. Scanning over all allowed values of $T_{\mathrm{ra}}$, the full range of masses and mixing angles for which we can obtain coherently oscillating relaxion DM is indicated by the black-framed regions in Fig. 3. We obtain two separated islands of viable parameter space, one at low masses, $10^{-11} \mathrm{eV} \lesssim m_{\phi} \lesssim 10^{-6} \mathrm{eV}$, with mixing angles around $10^{-23} \lesssim \sin \theta_{h \phi} \lesssim 10^{-18}$, and another island at high masses, $10^{-2} \mathrm{eV} \lesssim m_{\phi} \lesssim 1 \mathrm{eV}$, with a narrow range of mixing angles around $10^{-14} \lesssim \sin \theta_{h \phi} \lesssim 10^{-11}$. Note that, in the high-mass island, the coupling to dark photons is required to trap the relaxion, whereas in most of the lowmass island, relaxion DM can be realized without dark photon friction [6]. It shall, moreover, be emphasized that the low-mass DM regions in the minimal and dark photon scenario are separated in the reappearance temperature, since relaxion stopping via Hubble friction requires $m_{\phi} \lesssim \sqrt{8} \delta H_{\text {ra }}$, whereas dark photon production only occurs for $m_{\phi} \gtrsim \sqrt{10} \delta H_{\text {ra. }}$. See Appendix A for a detailed discussion on the minimal scenario.

\section{GRAVITATIONAL WAVES}

Let us now consider the stochastic GW background generated from anisotropies in the energy-momentum tensor of the dark photon produced during the postinflationary evolution of the relaxion. In particular, we focus here on the GWs sourced during the rolling of the relaxion between reheating and the EW phase transition. Further gravitational radiation can be generated when the relaxion oscillates around the minimum of its potential, as explored in Refs. [21-23,47-50] in the context of general ALP models, or in a confining phase transition that generates the backreaction potential [15]. In combination, the various sources may lead to an interesting and rich GW spectrum with multiple peaks.

The spectrum of a stochastic background of GWs is characterized by its fractional energy density, i.e., the energy density normalized to the critical value, $\rho_{c}^{0}=3 M_{\mathrm{Pl}}^{2} H_{0}^{2}$, per logarithmic frequency interval,

$$
\Omega_{\mathrm{GW}}(f)=\frac{1}{\rho_{c}^{0}} \frac{d \rho_{\mathrm{GW}}}{d \log f},
$$

where the total GW energy density is given by

$$
\rho_{\mathrm{GW}}=\frac{M_{\mathrm{Pl}}^{2}}{4}\left\langle\dot{h}_{i j} \dot{h}^{i j}\right\rangle=\int \frac{d f}{f} \frac{d \rho_{\mathrm{GW}}}{d \log f} .
$$

Here, $h_{i j}$ denotes the GW metric perturbations, and the dot indicates the derivative with respect to cosmic time $t$. Switching to conformal time $\tau, d t=a d \tau$, where $a$ is the scale factor of the Universe, the metric reads

$$
d s^{2}=a(\tau)\left\{d \tau^{2}-\left[\delta_{i j}+h_{i j}(\mathbf{x}, \tau)\right] d x^{i} d x^{j}\right\} .
$$

During radiation domination, the Einstein equations in the linear regime for the metric perturbations in momentum space using transverse-traceless gauge become

$$
\left(\partial_{\tau}^{2}+k^{2}\right) a(\tau) h_{i j}(\mathbf{k}, \tau)=\frac{2 a(\tau)}{M_{\mathrm{Pl}}^{2}} \Pi_{i j}(\mathbf{k}, \tau),
$$

where $k=|\mathbf{k}|$ is the comoving wave number. The anisotropic stress tensor $\Pi_{i j}$ relates to the energy-momentum 
tensor $T_{i j}$, via $\Pi_{i j}(\mathbf{k}, \tau)=\Lambda_{i j}^{a b}(\mathbf{k}) T_{a b}(\mathbf{k}, \tau)$, where $\Lambda_{i j}^{a b}=$ $P_{i}^{a} P_{j}^{b}-\frac{1}{2} P_{i j} P^{a b}$ with $P_{i j}=\delta_{i j}-k_{i} k_{j} / k^{2}$ being the projector that extracts the transverse and traceless part [1]. The equations of motion are then solved by (neglecting the $a^{\prime \prime}$ term, which vanishes in a radiation-dominated universe, i.e., for $a \propto \tau$ )

$$
\hat{h}_{i j}(\mathbf{k}, \tau)=\frac{2}{M_{\mathrm{Pl}}^{2}} \int^{\tau} d \tau^{\prime} \frac{a\left(\tau^{\prime}\right)}{a(\tau)} \hat{\Pi}_{i j}(\mathbf{k}, \tau) \mathcal{G}\left(k, \tau, \tau^{\prime}\right),
$$

where $\mathcal{G}\left(k, \tau, \tau^{\prime}\right)=\sin \left[k\left(\tau-\tau^{\prime}\right)\right] / k$ is the causal Green's function. We denote here the operator form of any quantity $Q$ by $\hat{Q}$.

\section{A. Gravitational wave production}

The GW energy density per logarithmic interval in the comoving momentum $k$ of a generic stochastic source at conformal time $\tau$ is given by [1]

$$
\begin{aligned}
\frac{d \rho_{\mathrm{GW}}}{d \log k}(k, \tau)= & \frac{k^{3}}{4 \pi^{2} M_{\mathrm{Pl}}^{2} a^{4}(\tau)} \int_{\tau_{i}}^{\tau} d \tau^{\prime} \int_{\tau_{i}}^{\tau} d \tau^{\prime} a\left(\tau^{\prime}\right) a\left(\tau^{\prime \prime}\right) \\
& \times \cos \left[k\left(\tau^{\prime}-\tau^{\prime \prime}\right)\right] \Pi^{2}\left(k, \tau^{\prime}, \tau^{\prime \prime}\right),
\end{aligned}
$$

where $\tau_{i}$ is the time at which the GW source starts operating and $\Pi^{2}\left(k, \tau^{\prime}, \tau^{\prime \prime}\right)$ is the unequal time correlator of the anisotropic stress, defined as $\left\langle 0\left|\hat{\Pi}^{a b}(\mathbf{k}, \tau) \hat{\Pi}_{a b}^{*}\left(\mathbf{k}^{\prime}, \tau^{\prime}\right)\right| 0\right\rangle=$ $(2 \pi)^{3} \delta\left(\mathbf{k}-\mathbf{k}^{\prime}\right) \Pi^{2}\left(k, \tau, \tau^{\prime}\right)$. In our case, the GWs are generated between reheating and reappearance; hence, $\tau_{i}=\tau_{\mathrm{rh}}$ and $\tau \leq \tau_{\mathrm{ra}}$. As the GWs produced before the relaxion reaches its terminal velocity will, however, be subdominant, we can take $\tau_{i}=\tau_{\mathrm{pp}}$, so to first approximation, the GW signature becomes independent of the temperature to which the Universe was reheated.

The dark photon anisotropic stress sourcing the GWs can be written in terms of the dark electric and magnetic fields as

$$
\begin{aligned}
\hat{\Pi}_{a b}(\mathbf{k}, \tau)= & -\frac{\Lambda_{a b}^{i j}(\mathbf{k})}{a^{2}(\tau)} \int \frac{d^{3} q}{(2 \pi)^{3}}\left[\hat{E}_{i}(\mathbf{q}, \tau) \hat{E}_{j}(\mathbf{k}-\mathbf{q}, \tau)\right. \\
& \left.+\hat{B}_{i}(\mathbf{q}, \tau) \hat{B}_{j}(\mathbf{k}-\mathbf{q}, \tau)\right] .
\end{aligned}
$$

Focusing on the dominant modes which have completed their phase of maximal tachyonic growth, $q \gtrsim r_{X}\left|\theta^{\prime}\right| / 2$, we find that GWs with momentum $k$ are dominantly produced at the time of reappearance. For frequencies below the peak, both dark photon modes in Eq. (33) have momenta close to the one that experiences maximal growth at reappearance, $|\mathbf{q}| \sim|\mathbf{k}-\mathbf{q}| \sim r_{X}\left|\theta_{\mathrm{ra}}^{\prime}\right| / 2$, whereas above the peak, one of the contributing modes must have a larger momentum and therefore have exited the tachyonic band before reappearance. Details of the calculation are deferred to Appendix C. The present-day GW spectrum can then be written as

$$
\Omega_{\mathrm{GW}}(f)=\Omega_{\mathrm{GW}}^{\text {peak }} \mathcal{S}_{\xi}\left(f / f_{\text {peak }}\right)
$$

with the peak frequency

$$
f_{\text {peak }}=\frac{k_{\mathrm{ra}}}{2 \pi a_{0}}=\frac{r_{X}\left|\theta_{\mathrm{ra}}^{\prime}\right|}{2 \pi a_{0}}=\frac{a_{\mathrm{ra}}}{a_{0}} \frac{\xi H_{\mathrm{ra}}}{2 \pi},
$$

where $k_{\text {ra }}$ is the mode that exits the tachyonic band at reappearance, and the peak amplitude

$$
\Omega_{\mathrm{GW}}^{\mathrm{peak}}=\frac{1}{\rho_{c}^{0}} \frac{a_{\mathrm{ra}}^{4}}{a_{0}^{4}} \frac{1}{M_{\mathrm{Pl}}^{2} H_{\mathrm{ra}}^{2}} \frac{\Lambda_{\mathrm{br}}^{8}}{4 r_{X}^{2}}=\frac{1}{\rho_{c}^{0}} \frac{a_{\mathrm{ra}}^{4}}{a_{0}^{4}} \frac{m_{\phi}^{4} f_{\phi}^{4}}{M_{\mathrm{Pl}}^{2} \xi^{2} H_{\mathrm{ra}}^{2}} .
$$

We used Eq. (13) here as well as $\xi \simeq 2 \delta r_{X}$ and $\Lambda_{\mathrm{br}}^{4}=$ $m_{\phi}^{2} f_{\phi}^{2} / \delta$ to recast Eqs. (35) and (36) into the estimations in Eqs. (1) and (2). The spectral shape $\mathcal{S}_{\xi}$ is given by

$$
\mathcal{S}_{\xi}(x)=\frac{1}{1+\frac{48}{5}(x-1)^{4}+\frac{19965}{256 \xi^{2}}\left(x^{-3}+3 x-4\right)} .
$$

Note that, similar to the oscillating axion case [21-23,47], we obtain a GW spectrum with an unpolarized lowfrequency tail and a chiral spectrum above the peak.

\section{B. GW detection}

Having predicted the GW spectrum generated by the relaxion dynamics, we can now evaluate its detectability in current and future experiments. A stochastic GW background can be detected in a given experiment if its signalto-noise ratio (SNR) $\varrho$ exceeds a threshold value $\varrho_{\text {thr }}$. The SNR is given by [51]

$$
Q^{2}=T_{\mathrm{obs}} \int_{f_{\min }}^{f_{\max }} d f\left[\frac{\Omega_{\mathrm{GW}}(f)}{\Omega_{n}(f)}\right]^{2},
$$

where $T_{\mathrm{obs}}$ is the period of observation, $\left(f_{\min }, f_{\max }\right)$ is the frequency range of the detector, and $\Omega_{n}(f)$ is the detector's noise spectrum converted to fractional energy density. For a cross-correlated measurement in a network of detectors, as, for instance, a pulsar timing array (PTA), the noise spectrum has to be replaced by the effective noise $\Omega_{\text {eff }}$ of the network (see Ref. [51] for further details), and the SNR is given by Eq. (38) with an additional factor of 2 .

We present here the projected sensitivities of the planned space-based Laser Interferometer Space Antenna (LISA) [52,53], the planned SKA [54] PTA observatory, ${ }^{6}$ and the proposed microhertz experiment $\mu$ Ares [56]. In addition, we evaluate current limits from the NANOGrav 11 year dataset [57]. Other GW observatories such as ground-based interferometers or potential LISA-successor experiments in

\footnotetext{
${ }^{6} \mathrm{We}$ assume here that the prospective foreground from supermassive black hole binaries can be subtracted. Further details on the sensitivities can be found in Ref. [55].
} 
the decihertz regime do not cover the frequency range in which a signal is expected in our scenario.

Recently, NANOGrav has further reported strong evidence for a common-spectrum stochastic process across the pulsars in their 12.5 year dataset [58], which might be due to a GW background. However, a GW detection could not be established yet, due to the lack of conclusive evidence regarding the interpulsar correlations of this process. Nonetheless, we also present here an interpretation of this signal as the GW background generated in our model, fitting our signal to the NANOGrav data based on the procedure outlined in Ref. [59].

Furthermore, GWs contribute to the total amount of radiation in the Universe and are therefore subject to constraints on $N_{\text {eff }}$. This sets an upper bound on the peak amplitude of the spectrum. Using Eq. (23), we obtain

$$
h^{2} \Omega_{\mathrm{GW}}^{\mathrm{peak}} \leq \frac{a_{\mathrm{rec}}^{4}}{a_{0}^{4}} \frac{7 \pi^{2}}{120}\left(\frac{4}{11}\right)^{\frac{4}{3}} \frac{T_{\mathrm{rec}}^{4}}{\rho_{c}^{0} / h^{2}} \frac{\Delta N_{\mathrm{eff}}}{\mathcal{I}_{\mathcal{S}}(\xi)} \approx 10^{-6}
$$

where $\mathcal{I}_{\mathcal{S}}(\xi)=\int d \log x \mathcal{S}_{\xi}(x) \simeq 0.8-1.5$ is the integral over the spectral shape and $T_{\text {rec }}$ is the photon temperature at recombination.

An even stronger bound is obtained from the contribution of the dark photons to $\Delta N_{\text {eff }}$. Since the GWs are sourced by dark photon anisotropies, this directly leads to an upper bound on the GW amplitude which is stronger than the one from the direct contribution of the GWs to $\Delta N_{\text {eff }}$. Plugging Eq. (24) into Eq. (36), we obtain

$$
h^{2} \Omega_{\mathrm{GW}}^{\mathrm{peak}} \lesssim 10^{-8}\left(\frac{g_{* \text {,ra }}}{106.75}\right)^{\frac{1}{3}}\left(\frac{25}{\xi}\right)^{2}\left(\frac{\Delta N_{\mathrm{eff}}^{X}}{0.52}\right)^{2},
$$

where the superscript $X$ indicates that this is the dark photon contribution to $\Delta N_{\text {eff }}$. A similar constraint on the GW amplitude was already observed in Ref. [59] in the audible axion scenario. There, it was noted that the parameter region that provides the best fit to the stochastic GW background that was potentially observed by the NANOGrav collaboration [58] is in tension with constraints on $\Delta N_{\text {eff }}$.

\section{DISCUSSION}

In Fig. 4, we show some example GW spectra. The corresponding parameter values are listed in Table I. The coupling to dark photons is set to $r_{X}=\xi /(2 \delta)$. In addition, the projected sensitivities of LISA (red), $\mu$ Ares (green), and SKA with an observation period of 5 years (turquoise) and 20 years (blue), represented by the corresponding powerlaw integrated sensitivity curves [51], are depicted as shaded regions. The current $95 \%$ upper limits from the 11 year NANOGrav dataset [57] are shown in purple. The example spectra $\mathrm{B}-\mathrm{D}$ are detectable with $\mu$ Ares, whereas the benchmark $A$ is accessible via SKA and is excluded by NANOGrav. The benchmark labeled " $*$ " shown in orange corresponds to our best fit to the NANOGrav 12.5 year dataset, as discussed further below.

At low frequencies, the GW spectra behave as approximately $f^{3}$, in accordance with the expectations based on causality arguments using that the anisotropic stress of a causal source cannot be correlated at scales above the horizon size at the time of production [1,60]. At high frequencies, the spectra fall like approximately $f^{-4}$, allowing a simple distinction from the much steeper falling GW background generated from oscillating [21-23,47] or rotating [61] axionlike fields. It should further be noted

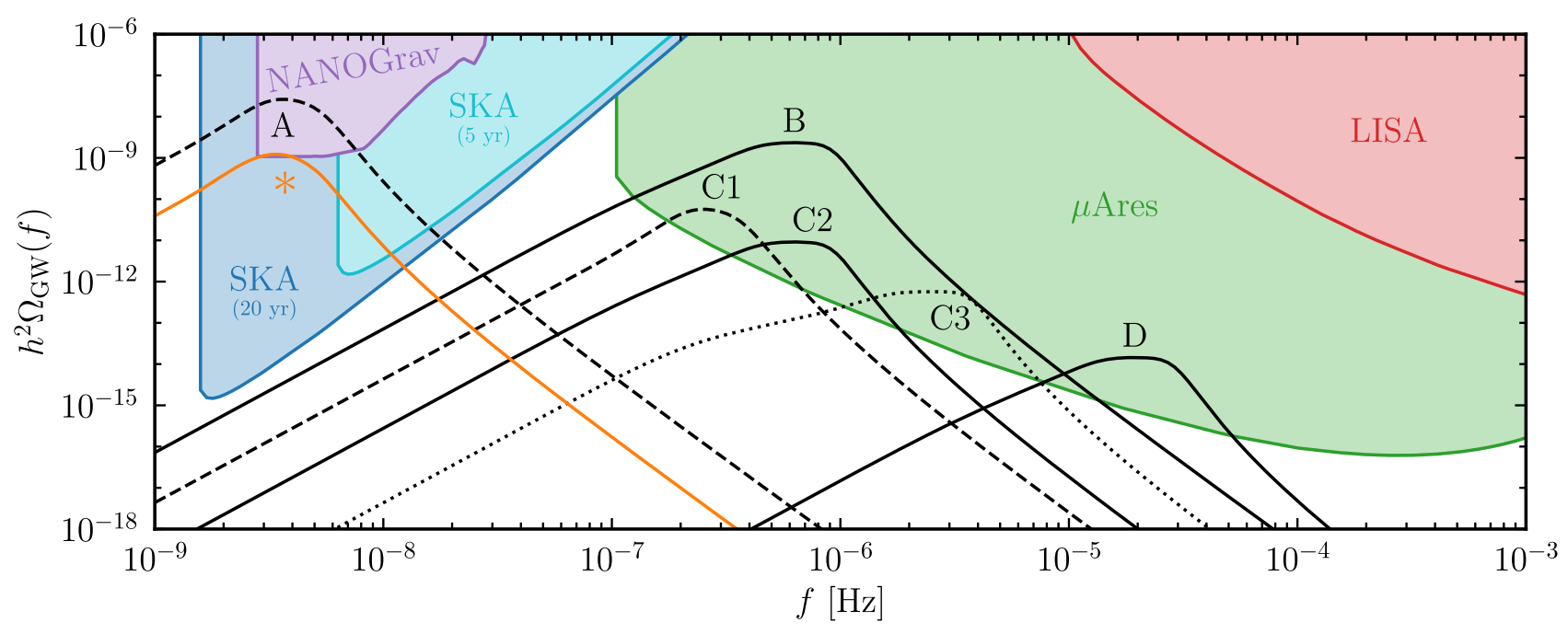

FIG. 4. GW spectra for the parameter points listed in Table I. The orange spectrum labeled " $*$ " corresponds to our best-fit to the NANOGrav 12.5 year dataset. The colored regions depict the projected power-law integrated sensitivity curves of LISA (red), $\mu$ Ares (green), and SKA (turquoise and blue, assuming 5 and 20 years of observation, respectively) and the current exclusion from the NANOGrav 11 year dataset (purple). 
TABLE I. Parameter values for the spectra shown in Fig. 4.

\begin{tabular}{lcccr}
\hline \hline & $m_{\phi}(\mathrm{eV})$ & $f_{\phi}(\mathrm{GeV})$ & $T_{\mathrm{ra}}(\mathrm{GeV})$ & $\xi$ \\
\hline $\mathrm{A}$ & $3 \times 10^{-9}$ & $10^{14}$ & 0.02 & 10 \\
$\mathrm{~B}$ & $2 \times 10^{-3}$ & $10^{12}$ & 1 & 25 \\
$\mathrm{C} 1$ & 5 & $10^{8}$ & 1 & 10 \\
$\mathrm{C} 2$ & $5 \times 10^{-3}$ & $10^{11}$ & 1 & 25 \\
$\mathrm{C} 3$ & $5 \times 10^{-6}$ & $10^{14}$ & 1 & 100 \\
$\mathrm{D}$ & 0.1 & $10^{12}$ & 30 & 25 \\
$*$ & $10^{-9}$ & $10^{14}$ & 0.02 & 10 \\
\hline \hline
\end{tabular}

that, when the peak position is fixed, changing $\xi$ barely affects the UV tail, while the IR tail goes as $\xi^{2}$ (cf. Fig. 9), potentially allowing one to disentangle the reappearance temperature and $\xi$ in the peak frequency Eq. (35) and thereby facilitating the determination of the relaxion parameters from a hypothetical observed signal. Larger values of $\xi$ further result in a flatter peak, although this may be an artifact of our analytic approximation; cf. Eq. (37).

The range of peak frequencies and amplitudes that can be attained for coherently oscillating relaxion DM is displayed in Fig. 5, considering the case when the relaxion starts oscillating at the time the wiggles reappear. The polygons are obtained from the allowed range for the cutoff $\Lambda$, for $T_{\text {ra }}$ between approximately $240 \mathrm{MeV}$ and $v_{H}$; cf. Fig. 2 . The solid and dashed lines assume $\xi=100$ and $\xi=10$, respectively. Peak positions inside the polygons can be realized. The sensitivities of $\mu$ Ares, LISA, and PTAs are again indicated as shaded regions. Note that the shading and solid lines correspond to the sensitivity for $\xi=100$. For $\xi=10$, the detection reach is degraded to the correspondingly colored dashed lines.

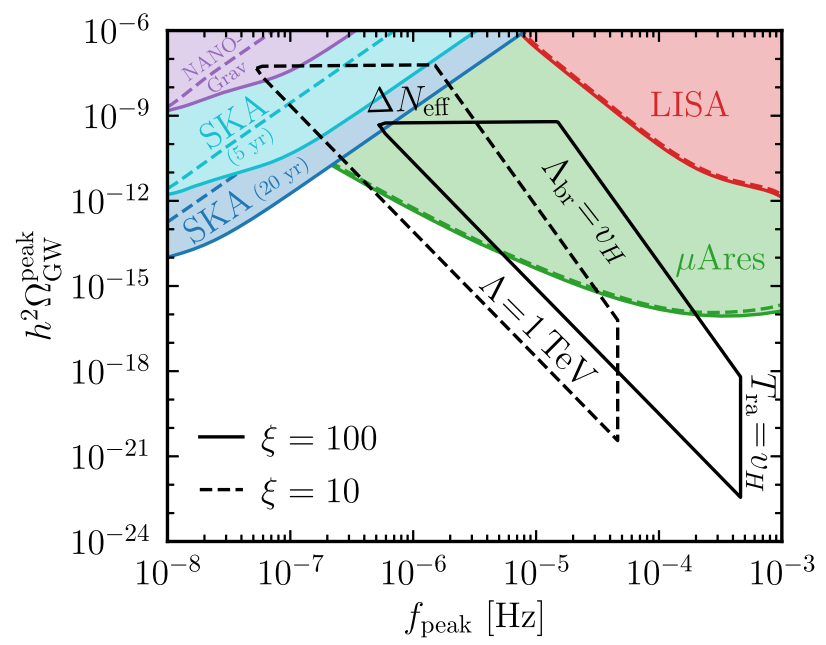

FIG. 5. Values of the peak frequency and amplitude of the GW spectrum which can be obtained in the relaxion DM scenario. The edges of the polygon correspond to the minimal and maximal amplitudes which can be obtained for $\xi=100$ (solid lines) and $\xi=10$ (dashed lines), considering the case when the relaxion starts to oscillate immediately after barrier reappearance.
For a large part of the peak frequencies and amplitudes that can be realized with relaxion DM, an observable signal is obtained, although mostly requiring futuristic spacebased interferometers such as $\mu$ Ares for observation. For low values of $\xi \sim 10$, a detection with SKA is possible. The present-day sensitivity of NANOGrav and expected reach of LISA are, however, insufficient for a detection. While NANOGrav is able to exclude parts of the parameter space if the DM assumption is relaxed (cf. benchmark $\mathrm{A}$ in Fig. 4), the sensitivity of LISA will remain insufficient even in this more general case.

We also show the GW sensitivity for relaxion DM as colored regions in Fig. 3, using the same coloring scheme as above. The (light) colored regions enclosed by the solid green and blue lines here indicate the relaxion masses and mixing angles for which, at least in a subrange of the reappearance temperatures in accordance with the constraints, an observable signal in $\mu$ Ares and SKA can be obtained. While $\mu$ Ares covers the full low-mass island as well as the range $m_{\phi} \lesssim 0.1 \mathrm{eV}$ in the high-mass island, the sensitivity of SKA is limited to slightly lower DM masses. Note that, as the GW spectrum strongly depends on the reappearance temperature, a nonobservation in these experiments would in most cases not rule out the colored parameter space, as a detection can be evaded by adjusting the reappearance temperature to shift the signal outside the experiment's reach. In the dark-green colored region bounded by the dotted lines, however, the generated stochastic GW background is observable in $\mu$ Ares for the full range of allowed reappearance temperatures, guaranteeing a detectable signal in this region. The temperature dependence of the relaxion constraints and GW limits can be seen explicitly in the animated version of the figure that can be found in the Supplemental Material [20].

Last but not least, let us now dismiss the assumption that the relaxion constitutes DM and return to Fig. 1, where we again indicate the parameter regions in which the GW background can be observed in $\mu$ Ares (green), NANOGrav (purple), or SKA (blue/turquoise) by the respective coloring. The colored regions respect the $f_{\phi}<M_{\mathrm{Pl}}$ constraint. If we allow for super-Planckian decay constant, the regions extend to the dotted lines.

Regarding the GW sensitivity, the reader needs to be aware that the figure shows the projection of a fourdimensional plot, as $T_{\text {ra }}$ and $f_{\phi}$ (or $\Lambda$ ) are not fixed. While red and orange shading marks the values of $m_{\phi}$ and $\sin \theta_{h \phi}$ for which it is not possible to evade the respective constraints by adjusting the remaining parameters (i.e., these colored regions are definitely excluded), the GW contours (blue, turquoise, purple, and green) correspond to the maximal reach of the respective experiments. They are obtained by taking the maximal SNR that can be achieved in each experiment for the given values of $m_{\phi}$ and $\sin \theta_{h \phi}$. Given the experimental sensitivities we assume here, a detection via GWs can be evaded in all of the viable 
parameter space. In particular, the purple coloring and lines do not indicate that the corresponding parameter points are excluded by NANOGrav data but that NANOGrav constrains the reappearance temperature (and the decay constant) in this region. The same also applies to the projections for SKA and $\mu$ Ares. Furthermore, although the sensitivities overlap in the plot, $\mu$ Ares and PTAs operate in different frequency regimes and are therefore typically sensitive to different ranges of $T_{\text {ra. }}$. Nonetheless, we can conclude that current and future GW experiments can potentially detect the stochastic GWs from relaxion stopping via dark photon emission and thereby constrain the parameter space. We again enclose an animated version of the figure in the Supplemental Material [20] available online, where the dependence on $T_{\text {ra }}$ (but not on $\Lambda$ ) is shown.

In addition to the current and prospective exclusion range, we also work out the parameter range in which our model can account for the potential GW signal recently observed in NANOGrav [58]. Fitting our spectrum to the data using the same procedure as in Ref. [59], where we keep $\xi$ fixed and only fit the peak frequency and amplitude, we obtain the best-fit points and the corresponding $1 \sigma$ and $2 \sigma$ contours shown in Fig. 6. We further indicate the minimal peak frequency dictated by the lower bound on the reappearance temperature, $T_{\mathrm{ra}} \gtrsim T_{\mathrm{BBN}}$, as well as the maximal peak amplitude consistent with the constraint on $\Delta N_{\text {eff }}$ by dotted lines. While these bounds exclude an explanation of the observed stochastic process in terms of our model for large values of $\xi \sim 100$ (green), we can reach into the $1 \sigma$ and $2 \sigma$ regions for intermediate $\xi \sim 25$ (orange), and for $\xi \sim 10$ (blue), we can account for the NANOGrav best-fit point.

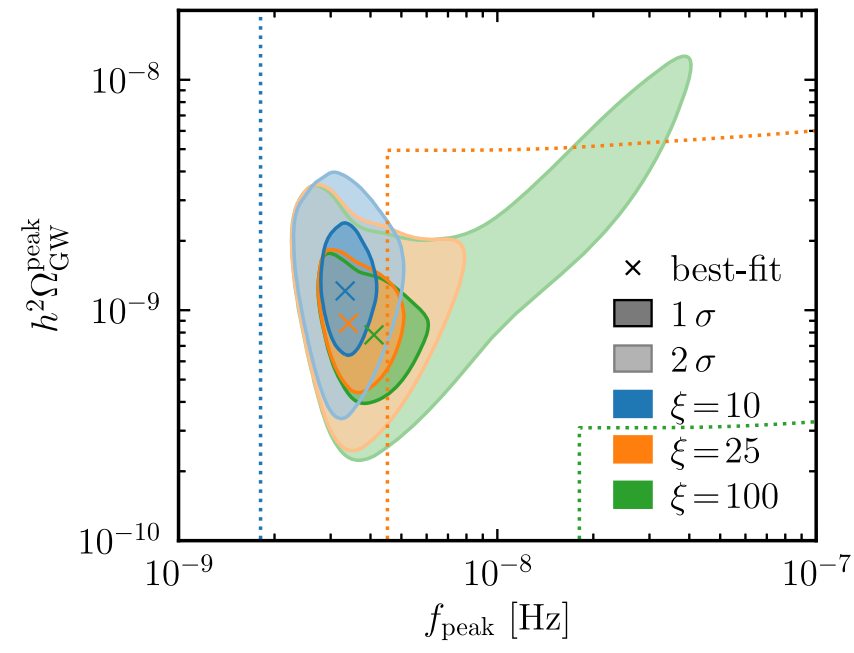

FIG. 6. Best-fit point (cross) to the NANOGrav 12.5 year data as well as $1 \sigma$ (dark) and $2 \sigma$ (light) regions, fixing $\xi$ to 10 (blue), 25 (orange), and 100 (green). The dotted lines enclose the region that can be obtained without being in tension with $\Delta N_{\text {eff }}$ or BBN at the respective value of $\xi$.
In the $\xi=10$ (lower) panel of Fig. 1, we indicate the values of $m_{\phi}$ and $\sin \theta_{h \phi}$ for which we can attain the best-fit point $\left(f_{\text {peak }}=3.3 \mathrm{nHz}, h^{2} \Omega_{\mathrm{GW}}^{\text {peak }}=1.2 \times 10^{-9}\right)$ by the gray shaded region. Note that, as we fix $\xi$ and $f_{\text {peak }}$, this also fixes the reappearance temperature to $T_{\mathrm{ra}} \sim 20 \mathrm{MeV}$, while the peak amplitude then fixes $f_{\phi}$ as a function of $m_{\phi}$. Hence, in the gray shaded region, $\Lambda$ can be adjusted within the constraints to obtain the respective value of the mixing angle. The best-fit spectrum for $\xi=10$ is also depicted in orange in Fig. 4.

\section{CONCLUSION}

In this work, we have considered the possibility of probing the relaxion, which was proposed to ameliorate the Higgs hierarchy problem, via gravitational waves. A coupling to dark photons tames the relaxion excursion after reheating and thus in turn opens up a large fraction of the parameter space which was excluded in the minimal scenario without dark photons. Furthermore, dark photon production after reheating can act as a source for the generation of a stochastic gravitational wave background. The gravitational waves are sourced by the anisotropies that are induced by the tachyonic production of dark photons between the electroweak phase transition and big bang nucleosynthesis. Hence, instead of the inflationary dynamics responsible for solving the hierarchy problem, we are probing here the late-time dynamics of the relaxion.

We have shown that this stochastic gravitational wave background can be probed by various current (NANOGrav) and future (SKA and $\mu$ Ares) gravitational wave detectors. In addition, we also highlight the parameter range in which our gravitational wave signal can account for the commonspectrum process observed in the most recent NANOGrav data [58]. Alongside the existing theoretical constraints, we have presented the relaxion parameter space which can be detected or excluded by the gravitational wave observatories in Fig. 1.

We find that the spectral shape of the gravitational wave signal in our model falls as the fourth power of the frequency above the peak, unlike a steeper falling gravitational wave signals generated by other axionlike field dynamics [21-23,61], whereas it behaves like $f^{3}$ in the infrared, as expected based on causality arguments. An observation of the spectrum in the range around the peak should allow for a determination of the reappearance temperature, while the amplitude can be used to determine the product of the relaxion mass and decay constant.

Furthermore, we have shown that the relaxion can constitute dark matter in the present Universe in the mass range of $10^{-11} \mathrm{eV} \lesssim m_{\phi} \lesssim 10^{-6} \mathrm{eV}$ and $10^{-2} \mathrm{eV} \lesssim m_{\phi} \lesssim 1 \mathrm{eV}$. While this scenario cannot be constrained with current NANOGrav data, most of the dark matter parameter space will be accessible by SKA and/or $\mu$ Ares in the future. Hence, with the advent of gravitational wave astronomy, we are now 
facing promising prospects for probing the relaxation of the electroweak scale via the stochastic gravitation wave background generated when stopping the relaxion, independently of whether the relaxion constitutes dark matter or not.

\section{ACKNOWLEDGMENTS}

We are grateful to Hyungjin Kim for collaboration at the early stages of this paper and participating in numerous vital discussions that seeded this work. We thank Edoardo Vitagliano for pointing out the updated fifth force bounds. The work of A. B. is supported by the Azrieli Foundation. The work of E. M. is supported by the Minerva Foundation. The work of G. P. is supported by grants from BSF-NSF, Friedrich Wilhelm Bessel research award, GIF, ISF, Minerva, Yeda-Sela-SABRA-WRC, and the Segre Research Award. The work of W. R. and P. S. is supported by the Deutsche Forschungsgemeinschaft (DFG), Project No. 438947057 and by the Cluster of Excellence "Precision Physics, Fundamental Interactions, and Structure of Matter" (PRISMA + EXC 2118/1) funded by the German Research Foundation (DFG) within the German Excellence Strategy (Project No. 39083149).

\section{APPENDIX A: MINIMAL RELAXION SCENARIO}

Here, we discuss the minimal relaxion scenario where a coupling to dark photons is absent. As discussed in Sec. II, due to the restoration of the EW symmetry, the relaxion starts rolling again if the reheating temperature is higher than the EW scale, $v_{H}$. To trap the relaxion in the minimum where it stopped during inflation, we need to require that it is displaced by less than $\Delta \theta \lesssim 2 \delta$, where $2 \delta$ is the separation between the minimum and the maximum [7]. In the absence of a dark photon coupling, the Hubble friction is sufficient to trap the relaxion if $m_{\phi} \lesssim \sqrt{8} \delta H_{\text {ra }}$, where $H_{\mathrm{ra}}$ is the Hubble scale at the time of barrier reappearance [6]. In combination with the constraints discussed in Eqs. (19) to (21), this trapping condition limits the mixing angle as

$$
\begin{aligned}
& \sin \theta_{h \phi} \gtrsim \frac{m_{\phi}^{3 / 2} \Lambda_{\mathrm{min}}}{m_{h}^{2}\left(2 H_{\mathrm{ra}}^{2}\right)^{1 / 4}}\left(\text { from } \Lambda \gtrsim \Lambda_{\mathrm{min}}\right), \\
& \sin \theta_{h \phi} \lesssim \frac{4 M_{\mathrm{Pl}} m_{\phi} H_{\mathrm{ra}}}{m_{h}^{2} v_{H}}\left(\text { from } f_{\phi} \lesssim M_{\mathrm{Pl}}\right), \\
& \sin \theta_{h \phi} \lesssim \frac{2^{\frac{5}{4}} v_{H} \sqrt{m_{\phi} H_{\mathrm{ra}}}}{m_{h}^{2}} \quad\left(\text { from } \Lambda_{\mathrm{br}} \lesssim v_{H}\right),
\end{aligned}
$$

within which the relaxion can be trapped by the Hubble friction. Moreover, the above equations restrict the relaxion mass for which the minimal scenario can be realized to $m_{\phi} \lesssim 2 \times 10^{-5} \mathrm{eV}$.
In addition to these constraints, for the relaxion not to overclose the Universe, we need to require $\Omega_{\phi} \lesssim \Omega_{\mathrm{DM}}$. This further constrains the range of the relaxion mass, and thus the minimal relaxion scenario can only be realized for

$$
m_{\phi} \lesssim 5 \times 10^{-8} \mathrm{eV}
$$

Figure 7 shows the available parameter space in the relaxion mass $m_{\phi}$ vs mixing angle $\sin \theta_{h \phi}$ plane. We assume here the minimal model without coupling to dark photons and do not require the relaxion to account for DM. Again, the red and orange shaded regions are excluded by the indicated constraints. The condition that the relaxion remains trapped in the same minimum in which it settled before reheating, $\Delta \theta \lesssim 2 \delta$, then additionally excludes the region shaded in blue, with only the white region remaining as viable parameter space. In the turquoise shaded region, fulfilling $\Delta \theta \lesssim 2 \delta$ without dark photons further leads to an overclosure of the Universe. The region in which the relaxion can constitute DM is enclosed by the dashed black contour. If the relaxion is coupled to a dark photon, we further open up the blue and turquoise shaded regions of the parameter space. With a dark photon, relaxion DM can be realized within the solid black contour (same as the black framed regions of Fig. 3). Note that the dark photon scenario can only be applied in the region below the gray dashed line. Above the line, the condition $H_{\mathrm{pp}}>H_{\mathrm{ra}}$ required for dark photon production is not satisfied (cf. the discussion in Sec. II C).

\section{APPENDIX B: DARK PHOTON SPECTRUM}

As discussed in Sec. II A, since $\theta^{\prime}>0$ in our case, positive-helicity dark photon modes with $k<k_{\mathrm{ex}}=r_{X} \theta^{\prime}$ are tachyonic and experience exponential growth, whereas modes outside the tachyonic band oscillate. In particular, the amplitude of the oscillating modes does not change in time. At any time, the peak of the dark photon spectrum is given by the mode that experiences maximal growth with $k=k_{\mathrm{m}}(\tau)=\left|r_{X} \theta^{\prime}\right| / 2$. We therefore take the ansatz

$X_{+}(k, \tau)= \begin{cases}\mathcal{A}_{k} \cos (k \tau-\xi) & \text { for } k_{\mathrm{m}}<k<k_{\mathrm{pp}}, \\ 0 & \text { otherwise }\end{cases}$

where we neglect the negative-helicity modes as well as all modes that did not experience maximal growth yet, since these are exponentially suppressed. We treat modes with $k_{\mathrm{ex}}<k<k_{\mathrm{m}}$ like the oscillating modes in order to include the peak of the spectrum in our estimate.

Neglecting the energy produced before particle production, the dark photon energy density is given by

$$
\rho_{X}=\int_{k_{\mathrm{m}}}^{k_{\mathrm{pp}}} \frac{d k k^{2}}{4 \pi^{2} a^{4}}\left(\left|X^{\prime}\right|^{2}+k^{2}|X|^{2}\right)=\int_{k_{\mathrm{m}}}^{k_{\mathrm{pp}}} \frac{d k}{k} \frac{\left|\mathcal{A}_{k}\right|^{2} k^{5}}{4 \pi^{2} a^{4}},
$$




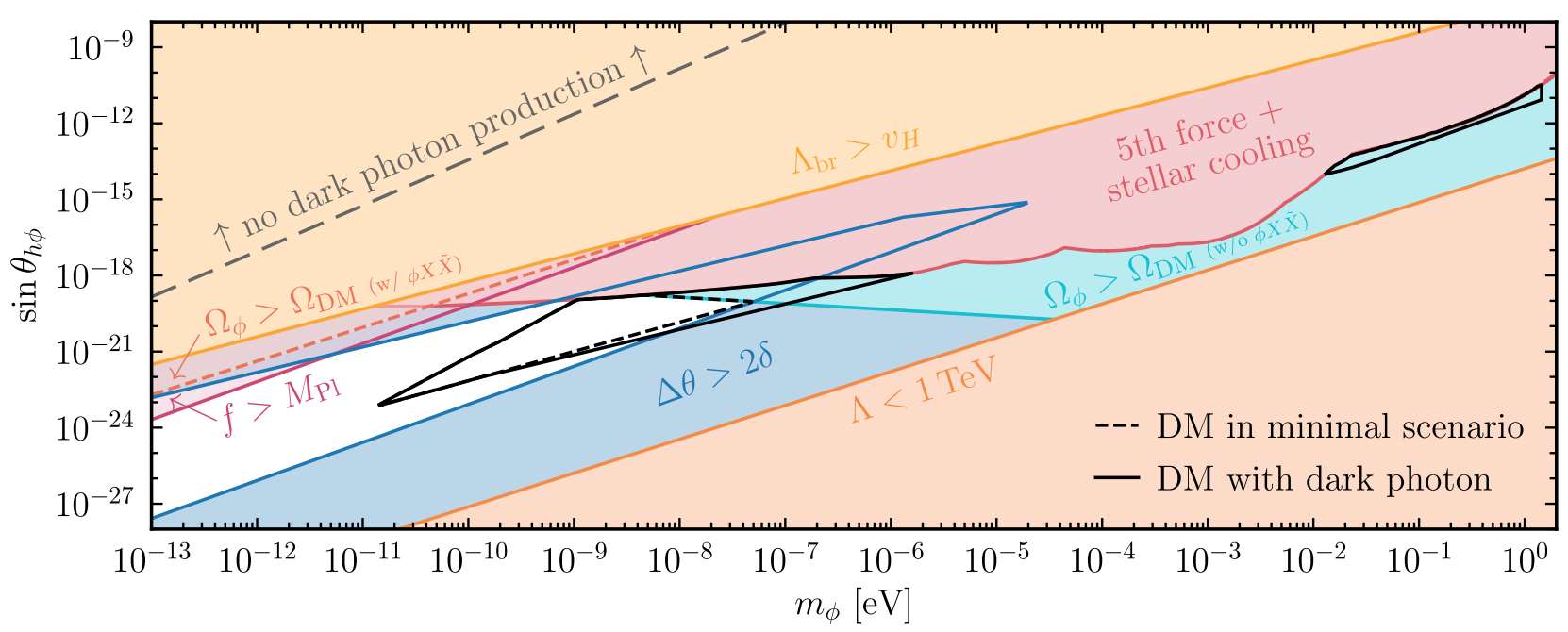

FIG. 7. Available parameter space in the minimal relaxion scenario when the reheating temperature is higher than the EW scale. The red and orange shaded regions are excluded by the indicated constraints or combinations thereof. The blue shaded region is further excluded if the relaxion is not coupled to the dark photon source term, leading to a field excursion of more than $2 \delta$. The dark photon production cannot occur in the region above the gray dashed line. Relaxion DM can be realized within the solid and dashed black contours, respectively, depending on whether a coupling to dark photons is assumed or not. In the turquoise parameter region, the condition $\Delta \theta \lesssim 2 \delta$ further leads to an overproduction of relaxion DM in the minimal scenario.

where $k_{\mathrm{pp}}=k_{\mathrm{ex}}\left(\tau_{\mathrm{pp}}\right)$ is the mode that exits the tachyonic band at particle production. On the other hand, we can determine the energy density spectrum of the dark photons from the relaxion energy density as

$$
\rho_{X}=-\int_{\tau_{\mathrm{pp}}}^{\tau} d \eta \frac{a^{4}(\eta)}{a^{4}(\tau)} \frac{\partial V}{\partial \theta} \frac{\partial \theta}{\partial \eta} \approx \int_{k_{\mathrm{m}}}^{k_{\mathrm{pp}}} \frac{d k}{k} \frac{k_{\mathrm{m}}^{4}}{k^{4}} \frac{\xi \Lambda_{\mathrm{br}}^{4}}{r_{X}}
$$

where we assumed that at each time energy is dominantly transferred into the maximally growing mode, which goes like $k_{\mathrm{m}} \propto 1 / \tau$. Hence, we can rewrite $\mathcal{A}_{k}=\mathcal{A}_{X} k^{-9 / 2}$ with

$$
\mathcal{A}_{X}=\frac{\pi}{2} \Lambda_{\mathrm{br}}^{2} \sqrt{\frac{\xi}{r_{X}}} a_{\mathrm{ra}}^{2} k_{\mathrm{ra}}^{2},
$$

where $k_{\mathrm{ra}}$ is the mode that exits the tachyonic band at reappearance, $k_{\mathrm{ra}}=\xi / \tau_{\mathrm{ra}}$. The dark photon spectrum therefore becomes

$X_{+}(k, \tau)= \begin{cases}\mathcal{A}_{X} k^{-\frac{9}{2}} \cos (k \tau-\xi) & \text { for } k_{\mathrm{m}}<k<k_{\mathrm{pp}} \\ 0 & \text { otherwise }\end{cases}$

To corroborate our estimation, we have simulated the dark photon and relaxion evolution after reheating, solving the equations of motion (7) and (8) numerically, using 5000 logarithmically spaced, discretized momenta. In Fig. 8, we present the result of the numeric simulation for the dark photon modes as a function of the momentum $k$. The simulation assumes $m_{\phi}=1 \mathrm{meV}, f_{\phi}=2.35 \times 10^{13} \mathrm{GeV}$, $\Lambda=100 \mathrm{TeV}$, and $\xi=77$. The value of $r_{X}$ has been determined numerically from Eq. (12) [6]. We show the spectrum at $a / a_{\mathrm{pp}}=4$ (red), 20 (green), 100 (orange), and 500 (blue), where the full and light colored lines correspond to the positive and negative helicity, respectively. As expected, the positive helicity modes dominate over the negative helicity by far. Furthermore, the amplitude for positive helicities indeed follows a $k^{-9 / 2}$ law [cf. Eq. (B5),

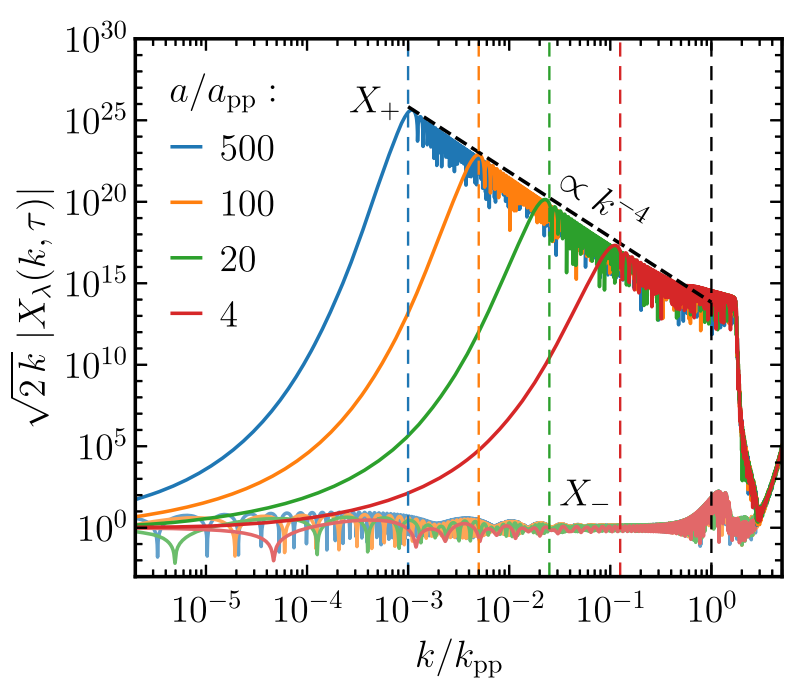

FIG. 8. Simulated (colored lines) and expected (dashed black line) amplitude of the dark photon modes at different times. The deep (light) colored lines correspond to the positive (negative) helicity. At each time, the expected peak momentum is indicated by the vertical dashed line in the corresponding color, whereas the black-dashed vertical line indicates the upper bound $k<k_{\mathrm{pp}}$ on the tachyonic dark photon momentum. 
i.e., $\sqrt{2 k}\left|X_{+}\right|$goes as $k^{-4}$ ] between the momentum $k_{\mathrm{pp}}$ that exits the tachyonic band at particle production and the peak momentum $k_{\mathrm{m}}(\tau)$ that experiences the largest growth rate at the respective time. The peak momenta and $k_{\mathrm{pp}}$ are indicated by the colored and black, dashed vertical lines, respectively.

\section{APPENDIX C: CALCULATION OF THE GRAVITATIONAL WAVE SPECTRUM}

The energy density of a stochastic GW background is given by

$$
\begin{aligned}
\rho_{\mathrm{GW}}(\mathbf{x}, \tau) & =\frac{M_{\mathrm{Pl}}^{2}}{4 a^{2}(\tau)}\left\langle\hat{h}_{a b}^{\prime}(\mathbf{x}, \tau) \hat{h}^{\prime a b}(\mathbf{x}, t)\right\rangle \\
& =\frac{M_{\mathrm{Pl}}^{2}}{8 \pi^{2} a^{2}(\tau)} \int d k k^{2} \mathcal{P}_{h^{\prime}}(k, \tau),
\end{aligned}
$$

where $\mathcal{P}_{h^{\prime}}$ is the power spectral density of $h_{a b}^{\prime}$ defined by $\left\langle\hat{h}^{\prime a b}(\mathbf{k}, \tau) \hat{h}_{a b}^{\prime *}\left(\mathbf{k}^{\prime}, \tau\right)\right\rangle=(2 \pi)^{3} \delta\left(\mathbf{k}-\mathbf{k}^{\prime}\right) \mathcal{P}_{h^{\prime}}(k, \tau)$. We hence obtain the GW spectrum

$$
\frac{d \rho_{\mathrm{GW}}}{d \log k}(k, \tau)=\frac{M_{\mathrm{Pl}}^{2} k^{3}}{8 \pi^{2} a^{2}(\tau)} \mathcal{P}_{h^{\prime}}(k, \tau)
$$

Using the solution Eq. (31), we obtain

$$
\begin{aligned}
\mathcal{P}_{h^{\prime}}(k, \tau)= & \frac{2}{M_{\mathrm{Pl}}^{4} a^{2}(\tau)} \int_{\tau_{\mathrm{pp}}}^{\tau} d \tau^{\prime} \int_{\tau_{\mathrm{pp}}}^{\tau} d \tau^{\prime \prime} \cos \left[k\left(\tau^{\prime}-\tau^{\prime \prime}\right)\right] \\
& \times a\left(\tau^{\prime}\right) a\left(\tau^{\prime \prime}\right) \Pi^{2}\left(k, \tau^{\prime}, \tau^{\prime \prime}\right)
\end{aligned}
$$

with the unequal time correlator again defined as $\left\langle 0\left|\hat{\Pi}^{a b}(\mathbf{k}, \tau) \hat{\Pi}_{a b}^{*}\left(\mathbf{k}^{\prime}, \tau^{\prime}\right)\right| 0\right\rangle=(2 \pi)^{3} \delta\left(\mathbf{k}-\mathbf{k}^{\prime}\right) \Pi^{2}\left(k, \tau, \tau^{\prime}\right)$, where we averaged over one period in $\tau$ to get rid of the $\cos \left[k\left(2 \tau-\tau^{\prime}-\tau^{\prime \prime}\right)\right]$ part.

Inserting Eqs. (10) and (33) into Eqs. (C2) and (C3) and rewriting the dark electric and magnetic fields in terms of the dark photon modes,

$$
\begin{gathered}
\hat{\mathbf{E}}(\mathbf{k}, \tau)=\sum_{\lambda} X_{\lambda}^{\prime}(k, \tau) \varepsilon_{\lambda}(\mathbf{k}) \hat{a}_{\lambda}(\mathbf{k})+\text { H.c. } \\
\hat{\mathbf{B}}(\mathbf{k}, \tau)=\sum_{\lambda} \lambda k X_{\lambda}(k, \tau) \varepsilon_{\lambda}(\mathbf{k}) \hat{a}_{\lambda}(\mathbf{k})+\text { H.c. }
\end{gathered}
$$

we obtain the spectrum at the time of reappearance as

$$
\begin{aligned}
\frac{d \rho_{\mathrm{GW}}}{d \log k}= & \frac{k^{3}}{2 \pi^{2} M_{\mathrm{Pl}}^{2} a_{\mathrm{ra}}^{4}} \int \frac{d^{3} q}{(2 \pi)^{3}}\left|\Sigma_{a b}(\mathbf{k}, \mathbf{q})\right|^{2} \\
& \times\left(\left|I_{c}(\mathbf{k}, \mathbf{q})\right|^{2}+\left|I_{s}(\mathbf{k}, \mathbf{q})\right|^{2}\right),
\end{aligned}
$$

where we used $\left[\hat{a}_{\lambda}(\mathbf{k}), \hat{a}_{\lambda^{\prime}}^{\dagger}\left(\mathbf{k}^{\prime}\right)\right]=(2 \pi)^{3} \delta_{\lambda \lambda^{\prime}} \delta\left(\mathbf{k}-\mathbf{k}^{\prime}\right)$ and the invariance of the integrand upon interchanging $\mathbf{q} \rightarrow \mathbf{k}-\mathbf{q}$. We only considered here the positive helicity modes and defined $\Sigma_{a b}(\mathbf{k}, \mathbf{q})=\Lambda_{a b}^{i j}(\mathbf{k}) \varepsilon_{i}^{+}(\mathbf{q}) \varepsilon_{j}^{+}(\mathbf{k}-\mathbf{q})$ as well as

$$
I_{c / s}(\mathbf{k}, \mathbf{q})=-\int_{\tau_{\mathrm{pp}}}^{\tau_{\mathrm{ra}}} \frac{d \tau}{a(\tau)}\left\{\begin{array}{l}
\cos (k \tau) \\
\sin (k \tau)
\end{array}\right\} \chi(q, l, \tau)
$$

with $\quad \chi(\mathbf{k}, \mathbf{q}, \tau)=X_{+}^{\prime}(q, \tau) X_{+}^{\prime}(l, \tau)+q X_{+}(q, \tau) l X_{+}(l, \tau)$ and $l=|\mathbf{k}-\mathbf{q}|$. Using the dark photon spectrum Eq. (B5), we obtain

$$
\chi(q, l, \tau)=\frac{\mathcal{A}_{X}^{2} \cos [(q-l) \tau]}{(q l)^{\frac{7}{2}}}
$$

for $\min (q, l)>k_{\mathrm{m}}(\tau)$.

Expressing the momenta in terms of $k_{\text {ra }}$, we can rewrite the energy density as

$$
\begin{aligned}
\frac{d \rho_{\mathrm{GW}}}{d \log k}= & \frac{\Lambda_{\mathrm{br}}^{8} \xi^{2}}{r_{X}^{2} H_{\mathrm{ra}}^{2} M_{\mathrm{Pl}}^{2}} x^{3} \int_{\frac{1}{2}}^{\infty} d r \int_{-1}^{1} d \cos \theta \frac{\left|\Sigma_{a b}(x, r, \cos \theta)\right|^{2}}{512 r^{5} s^{7}} \\
& \times\left(\left|\tilde{I}_{c}(x, r, s, \xi)\right|^{2}+\left|\tilde{I}_{s}(x, r, s, \xi)\right|^{2}\right) \Theta(s-1 / 2),
\end{aligned}
$$

where the remaining integrals only depend on $x=k / k_{\text {ra }}$ and $\xi$. We defined here $r=q / k_{\mathrm{ra}}$ and $s=l / k_{\mathrm{ra}}$. The polarization part is given by (see, e.g., Ref. [21] for further details)

$$
\left|\Sigma_{a b}\right|^{2}=\sum_{\lambda= \pm}\left[\frac{1+\lambda \cos \theta}{2}\right]^{2}\left[\frac{1+\lambda \frac{x-r \cos \theta}{s}}{2}\right]^{2},
$$

where $\lambda$ now denotes the GW helicity, and the time integrals evaluate to

$\tilde{I}_{c / s}=\left\{\begin{array}{l}\operatorname{Ci}[(x+r-s) \xi]-\operatorname{Ci}\left[\frac{(x+r-s) \xi}{2 \min (r, s)}\right]+(r \leftrightarrow s) \\ \operatorname{Si}[(x+r-s) \xi]-\operatorname{Si}\left[\frac{(x+r-s) \xi}{2 \min (r, s)}\right]+(r \leftrightarrow s),\end{array}\right.$

where $\mathrm{Ci}$ and $\mathrm{Si}$ are the cosine and sine integral function,

$$
\operatorname{Ci}(z)=\int_{z}^{\infty} d t \frac{\cos t}{t}, \quad \operatorname{Si}(z)=\int_{0}^{z} d t \frac{\sin t}{t} .
$$

The GW spectrum can now be obtained by evaluating Eq. (C8) numerically. In this paper, we, however, use an analytic approximation to the spectrum based on the amplitude at the peak and the asymptotic behavior in the UV and IR.

Since the GW momentum is given by the sum of two dark photon momenta, $\mathbf{k}=\mathbf{q}+\mathbf{l}$, and since the time integrals are dominated by the late-time behavior, the peak of the GW spectrum will roughly be given by twice the peak momentum of the dark photon spectrum at reappearance; i.e., we take $k_{\text {peak }}=k_{\text {ra. }}$. As the arguments of the 
cosine and sine integrals in Eq. (C10) are proportional to $\xi \sim \mathcal{O}(10-100)$, we can expand for large $\xi$. Assuming that the cosine and sine terms remaining in the expansion oscillate quickly and therefore average to zero, the corresponding amplitude for $x=1$ then evaluates to

$$
\frac{d \rho_{\mathrm{GW}}^{\mathrm{peak}}}{d \log k}=\left[\frac{1}{4 \xi^{2}}+\mathcal{O}\left(\xi^{-4}\right)\right] \frac{\xi^{2}}{r_{X}^{2}} \frac{\Lambda_{\mathrm{br}}^{8}}{H_{\mathrm{ra}}^{2} M_{\mathrm{Pl}}^{2}} .
$$

For $k \gg k_{\text {ra }}$, we have $x+r-s=r(1+\cos \theta)+\mathcal{O}\left(x^{-1}\right)$ and $x-r+s=2 x+\mathcal{O}(1)$. The $(r \leftrightarrow s)$ part of Eq. (C10) is hence suppressed by $1 / x$, and only the first two terms contribute. Again expanding for large $\xi$, we obtain that the spectrum behaves as

$$
\frac{d \rho_{\mathrm{GW}}^{\mathrm{UV}}}{d \log k}=\left[\frac{5}{192 \xi^{2}}+\mathcal{O}\left(\xi^{-4}\right)\right] \frac{\xi^{2}}{r_{X}^{2}} \frac{\Lambda_{\mathrm{br}}^{8}}{H_{\mathrm{ra}}^{2} M_{\mathrm{Pl}}^{2}} \frac{k_{\mathrm{ra}}^{4}}{k^{4}}
$$

in the UV. For $k \ll k_{\mathrm{ra}}$, on the other hand, the arguments of the cosine and sine integrals become proportional to $x \pm r \mp s=x(1 \pm \cos \theta)+\mathcal{O}\left(x^{2}\right)$, so we can expand the integrals for low arguments, yielding the IR behavior

$$
\frac{d \rho_{\mathrm{GW}}^{\mathrm{IR}}}{d \log k}=\frac{64}{19965} \frac{\xi^{2}}{r_{X}^{2}} \frac{\Lambda_{\mathrm{br}}^{8}}{H_{\mathrm{ra}}^{2} M_{\mathrm{Pl}}^{2}} \frac{k^{3}}{k_{\mathrm{ra}}^{3}} .
$$

As can be seen from Eq. (C9), at low momentum, $x \ll 1$, where we have $s \sim r$, both GW helicities contribute equally, whereas at high momentum, where $x \gg 1$ and hence $s \sim x$, the negative helicity is suppressed by a factor of $1 / k^{2}$ compared to the positive one.

Our approximation to the full spectrum is now obtained by combining these results inversely. Neglecting GW production after reappearance, the energy density will subsequently simply redshift as $\rho_{\mathrm{GW}} \sim a^{-4}$, so the spectrum today as a function of the comoving wave number $k$ becomes

$$
\begin{aligned}
\Omega_{\mathrm{GW}}(k)= & \frac{1}{\rho_{c}^{0}}\left(\frac{a_{\mathrm{ra}}}{a_{0}}\right)^{4} \frac{1}{M_{\mathrm{Pl}}^{2} H_{\mathrm{ra}}^{2}} \frac{\Lambda_{\mathrm{br}}^{8}}{4 r_{X}^{2}}\left\{1+\frac{19965}{256 \xi^{2}}\left[\left(\frac{k_{\mathrm{ra}}^{3}}{k^{3}}-1\right)\right.\right. \\
& \left.\left.+3\left(\frac{k}{k_{\mathrm{ra}}}-1\right)\right]+\frac{48}{5}\left(\frac{k}{k_{\mathrm{ra}}}-1\right)^{4}\right\}^{-1}, \quad(\mathrm{C} 15)
\end{aligned}
$$

where the first term in the curly bracket reproduces our estimate for the peak amplitude; the last term and the first part of the second term reproduce the spectrum in the IR and UV, respectively; and the remaining term in the square bracket renders $k=k_{\text {ra }}$ an extremum of the spectrum. Further noting that frequency $f$ and comoving wave number $k$ are related via $k d \tau=\frac{k}{a} d t=2 \pi f d t$, we end up with the present-day spectrum in Eqs. (34) to (37).

\footnotetext{
${ }^{7}$ The analytic integration actually yields $\frac{278880340+44998983 \log 3}{1254113280} \approx$ 0.262 instead of $1 / 4$.
}

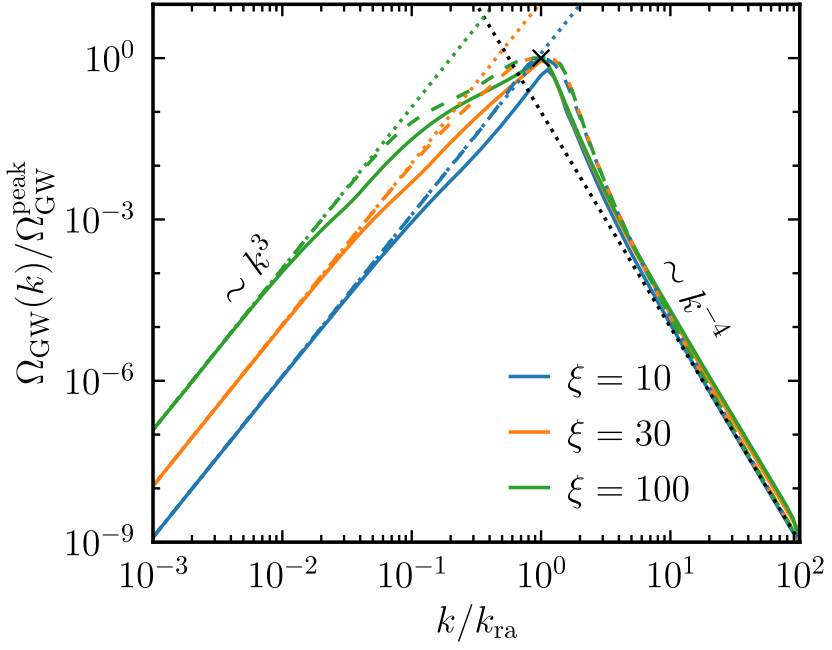

FIG. 9. Spectral shape of the stochastic GW background for $\xi=10$ (blue), 30 (orange), and 100 (green). The solid lines correspond to the numeric calculation, whereas the dashed ones are the analytic approximation. The dotted lines indicate the IR and UV behavior, and the black cross marks our estimate of the peak amplitude.

In Fig. 9, we compare the numerical result of the spectral shape based on Eq. (C8) (solid lines) to the analytic approximation in Eq. (C15) (dashed lines) for different values of $\xi$. In addition, the UV and IR behavior, Eqs. (C13) and (C14) as well as the peak amplitude Eq. (C12) are indicated by dotted lines and a cross, respectively. Our approximation agrees well with the numeric result.

Finally, Fig. 10 shows the GW spectrum from our numeric simulation (cf. Fig. 8), assuming a reappearance

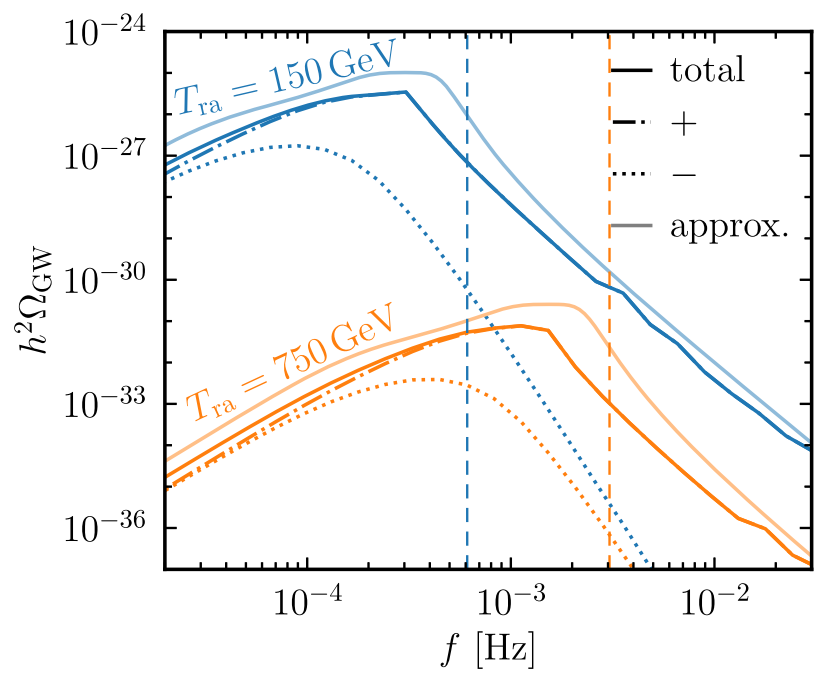

FIG. 10. Simulation (full colors) and analytic approximation (light colors) of the GW spectrum for a reappearance temperature of $T_{\text {ra }}=150 \mathrm{GeV}$ (blue) and $750 \mathrm{GeV}$ (orange). The vertical dashed lines indicate the expected peak frequency, whereas the dot-dashed and dotted curves correspond to the positive and negative helicity contributions to the simulated spectrum. 
temperature of $T_{\text {ra }}=150 \mathrm{GeV}$ (blue) and $750 \mathrm{GeV}$ (orange), corresponding to $a / a_{\mathrm{ra}}=500$ and $a / a_{\mathrm{ra}}=100$, respectively. We see that the GW background is unpolarized below the peak, whereas above the peak the positive polarization (dot-dashed) dominates over the negative one (dotted). Our estimates based on Eq. (C15) (light lines) overestimate the simulated spectra by an $\mathcal{O}(1-10)$ factor.
[1] C. Caprini and D. G. Figueroa, Cosmological backgrounds of gravitational waves, Classical Quantum Gravity 35, 163001 (2018).

[2] C. Caprini et al., Detecting gravitational waves from cosmological phase transitions with LISA: An update, J. Cosmol. Astropart. Phys. 03 (2020) 024.

[3] P. W. Graham, D. E. Kaplan, and S. Rajendran, Cosmological Relaxation of the Electroweak Scale, Phys. Rev. Lett. 115, 221801 (2015).

[4] D. S. Salopek, J. R. Bond, and J. M. Bardeen, Designing density fluctuation spectra in inflation, Phys. Rev. D 40, 1753 (1989).

[5] D. Bödeker and W. Buchmüller, Baryogenesis from the weak scale to the grand unification scale, arXiv:2009.07294.

[6] A. Banerjee, H. Kim, and G. Perez, Coherent relaxion dark matter, Phys. Rev. D 100, 115026 (2019).

[7] A. Banerjee, H. Kim, O. Matsedonskyi, G. Perez, and M. S. Safronova, Probing the relaxed relaxion at the luminosity and precision frontiers, J. High Energy Phys. 07 (2020) 153.

[8] K. Choi and S. H. Im, Constraints on relaxion windows, J. High Energy Phys. 12 (2016) 093.

[9] T. Flacke, C. Frugiuele, E. Fuchs, R. S. Gupta, and G. Perez, Phenomenology of relaxion-Higgs mixing, J. High Energy Phys. 06 (2017) 050.

[10] K. Choi, H. Kim, and T. Sekiguchi, Dynamics of the cosmological relaxation after reheating, Phys. Rev. D 95, 075008 (2017).

[11] A. Hook and G. Marques-Tavares, Relaxation from particle production, J. High Energy Phys. 12 (2016) 101.

[12] N. Fonseca, E. Morgante, R. Sato, and G. Servant, Axion fragmentation, J. High Energy Phys. 04 (2020) 010.

[13] N. Fonseca, E. Morgante, R. Sato, and G. Servant, Relaxion fluctuations (self-stopping relaxion) and overview of relaxion stopping mechanisms, J. High Energy Phys. 05 (2020) 080; J. High Energy Phys. 01 (2021) 012(E).

[14] S.-J. Wang, Paper-boat relaxion, Phys. Rev. D 99, 095026 (2019).

[15] D. Barducci, E. Bertuzzo, and M. Arteaga-Tupia, Gravitational tests of electroweak relaxation, J. High Energy Phys. 07 (2021) 119.

[16] S. Y. Khlebnikov and I. I. Tkachev, Relic gravitational waves produced after preheating, Phys. Rev. D 56, 653 (1997).

[17] R. Easther and E. A. Lim, Stochastic gravitational wave production after inflation, J. Cosmol. Astropart. Phys. 04 (2006) 010.

[18] J.-F. Dufaux, G. Felder, L. Kofman, and O. Navros, Gravity waves from tachyonic preheating after hybrid inflation, J. Cosmol. Astropart. Phys. 03 (2009) 001.
[19] P. Adshead, J. T. Giblin, and Z. J. Weiner, Gravitational waves from gauge preheating, Phys. Rev. D 98, 043525 (2018).

[20] See Supplemental Material at http://link.aps.org/supplemental/ 10.1103/PhysRevD.104.055026 for animated versions of Figs. 1 and 3, showing the dependence of the constraints and the GW sensitivity on the reappearance temperature.

[21] C. S. Machado, W. Ratzinger, P. Schwaller, and B. A. Stefanek, Audible axions, J. High Energy Phys. 01 (2019) 053.

[22] C. S. Machado, W. Ratzinger, P. Schwaller, and B. A. Stefanek, Gravitational wave probes of axionlike particles, Phys. Rev. D 102, 075033 (2020).

[23] W. Ratzinger, P. Schwaller, and B. A. Stefanek, Gravitational waves from an axion-dark photon system: A lattice study, SciPost Phys. 11, 001 (2021).

[24] K. Choi, H. Kim, and S. Yun, Natural inflation with multiple sub-Planckian axions, Phys. Rev. D 90, 023545 (2014).

[25] K. Choi and S. H. Im, Realizing the relaxion from multiple axions and its UV completion with high scale supersymmetry, J. High Energy Phys. 01 (2016) 149.

[26] D. E. Kaplan and R. Rattazzi, Large field excursions and approximate discrete symmetries from a clockwork axion, Phys. Rev. D 93, 085007 (2016).

[27] G. F. Giudice and M. McCullough, A clockwork theory, J. High Energy Phys. 02 (2017) 036.

[28] N. Aghanim et al. (Planck Collaboration), Planck 2018 results. VI. Cosmological parameters, Astron. Astrophys. 641, A6 (2020).

[29] E. W. Kolb and M. S. Turner, The Early Universe, Frontiers in Physics, Vol. 69 (Westview Press, 1990).

[30] B. Audren, J. Lesgourgues, G. Mangano, P. D. Serpico, and T. Tram, Strongest model-independent bound on the lifetime of Dark Matter, J. Cosmol. Astropart. Phys. 12 (2014) 028.

[31] P. Agrawal, G. Marques-Tavares, and W. Xue, Opening up the QCD axion window, J. High Energy Phys. 03 (2018) 049.

[32] N. Kitajima, T. Sekiguchi, and F. Takahashi, Cosmological abundance of the QCD axion coupled to hidden photons, Phys. Lett. B 781, 684 (2018).

[33] N. Kitajima, J. Soda, and Y. Urakawa, Nano-Hz GravitationalWave Signature from Axion Dark Matter, Phys. Rev. Lett. 126, 121301 (2021).

[34] C. A. J. O'Hare and E. Vitagliano, Cornering the axion with $C P$-violating interactions, Phys. Rev. D 102, 115026 (2020).

[35] W.-H. Tan et al., Improvement for Testing the Gravitational Inverse-Square Law at the Submillimeter Range, Phys. Rev. Lett. 124, 051301 (2020).

[36] J. Bergé, P. Brax, G. Métris, M. Pernot-Borràs, P. Touboul, and J.-P. Uzan, MICROSCOPE Mission: First Constraints 
on the Violation of the Weak Equivalence Principle by a Light Scalar Dilaton, Phys. Rev. Lett. 120, 141101 (2018).

[37] Y. J. Chen, W. K. Tham, D. E. Krause, D. López, E. Fischbach, and R. S. Decca, Stronger Limits on Hypothetical Yukawa Interactions in the 30-8000 nm Range, Phys. Rev. Lett. 116, 221102 (2016).

[38] D. J. Kapner, T. S. Cook, E. G. Adelberger, J. H. Gundlach, B. R. Heckel, C. D. Hoyle, and H. E. Swanson, Tests of the Gravitational Inverse-Square Law Below the Dark-Energy Length Scale, Phys. Rev. Lett. 98, 021101 (2007).

[39] S. Schlamminger, K. Y. Choi, T. A. Wagner, J. H. Gundlach, and E. G. Adelberger, Test of the Equivalence Principle Using a Rotating Torsion Balance, Phys. Rev. Lett. 100, 041101 (2008).

[40] J. K. Hoskins, R. D. Newman, R. Spero, and J. Schultz, Experimental tests of the gravitational inverse square law for mass separations from 2 to $105 \mathrm{~cm}$, Phys. Rev. D32,3084(1985).

[41] J. A. Grifols, E. Masso, and S. Peris, Energy loss from the Sun and RED giants: Bounds on short range baryonic and leptonic forces, Mod. Phys. Lett. A 04, 311 (1989).

[42] D. Cadamuro and J. Redondo, Cosmological bounds on pseudo Nambu-Goldstone bosons, J. Cosmol. Astropart. Phys. 02 (2012) 032.

[43] G. Raffelt, Limits on a $C P$-violating scalar axion-nucleon interaction, Phys. Rev. D 86, 015001 (2012).

[44] E. Hardy and R. Lasenby, Stellar cooling bounds on new light particles: Plasma mixing effects, J. High Energy Phys. 02 (2017) 033.

[45] R. Budnik, O. Davidi, H. Kim, G. Perez, and N. Priel, Searching for a solar relaxion or scalar particle with XENON1T and LUX, Phys. Rev. D 100, 095021 (2019).

[46] A. G. Riess et al., New parallaxes of galactic cepheids from spatially scanning the Hubble space telescope: Implications for the Hubble constant, Astrophys. J. 855, 136 (2018).

[47] B. Salehian, M. A. Gorji, S. Mukohyama, and H. Firouzjahi, Analytic study of dark photon and gravitational wave production from axion, J. High Energy Phys. 05 (2021) 043.

[48] J. Soda and Y. Urakawa, Cosmological imprints of string axions in plateau, Eur. Phys. J. C 78, 779 (2018).
[49] N. Kitajima, J. Soda, and Y. Urakawa, Gravitational wave forest from string axiverse, J. Cosmol. Astropart. Phys. 10 (2018) 008.

[50] A. Chatrchyan and J. Jaeckel, Gravitational waves from the fragmentation of axion-like particle dark matter, J. Cosmol. Astropart. Phys. 02 (2021) 003.

[51] E. Thrane and J. D. Romano, Sensitivity curves for searches for gravitational-wave backgrounds, Phys. Rev. D 88, 124032 (2013).

[52] P. Amaro-Seoane et al. (LISA Collaboration), Laser interferometer space antenna, arXiv:1702.00786.

[53] T. Robson, N. J. Cornish, and C. Liu, The construction and use of LISA sensitivity curves, Classical Quantum Gravity 36, 105011 (2019).

[54] G. Janssen et al., Gravitational wave astronomy with the SKA, Proc. Sci., AASKA14 (2015) 037 [arXiv:1501.00127].

[55] M. Breitbach, J. Kopp, E. Madge, T. Opferkuch, and P. Schwaller, Dark, cold, and noisy: Constraining secluded hidden sectors with gravitational waves, J. Cosmol. Astropart. Phys. 07 (2019) 007.

[56] A. Sesana et al., Unveiling the gravitational universe at $\mu-\mathrm{Hz}$ frequencies, arXiv:1908.11391.

[57] Z. Arzoumanian et al. (NANOGRAV Collaboration), The NANOGrav 11 year data set: Pulsar-timing constraints on the stochastic gravitational-wave background, Astrophys. J. 859, 47 (2018).

[58] Z. Arzoumanian et al. (NANOGrav Collaboration), The NANOGrav $12.5 \mathrm{yr}$ data set: Search for an isotropic stochastic gravitational-wave background, Astrophys. J. Lett. 905, L34 (2020).

[59] W. Ratzinger and P. Schwaller, Whispers from the dark side: Confronting light new physics with NANOGrav data, SciPost Phys. 10, 047 (2021).

[60] C. Caprini, R. Durrer, T. Konstandin, and G. Servant, General properties of the gravitational wave spectrum from phase transitions, Phys. Rev. D 79, 083519 (2009).

[61] R. T. Co, K. Harigaya, and A. Pierce, Gravitational waves and dark photon dark matter from axion rotations arXiv: 2104.02077. 